\title{
CHAPITRE 5 \\ ASPECTS GÉNÉTIQUES DE LA CONSTITUTION DE POPULATIONS D'ÉLEVAGE DESTINÉES AU REPEUPLEMENT
}

\section{B. Chevassus}

Laboratoire de Génétique des poissons

I.N.R.A. - 78350 JOUY-EN-JOSAS (FRANCE)

\begin{abstract}
Le développement d'une politique de repeuplement implique la création et l'entretien de stocks de reproducteurs en captivité. L'intervention de la génétique dans ce domaine est généralement envisagée sous l'angle de l'amélioration des performances, par sélection, croisement ou manipulations diverses. Par contre, la nécessité de mettre en place une gestion génétique des stocks, même en l'absence d'objectifs d'amélioration clairement identifiés, est souvent méconnue. II nous a donc semblé nécessaire de développer ce thème, en montrant en particulier en quoi divers aspects de la génétique peuvent intervenir dans la création de ces stocks.

Deux cas seront distingués:

- l'échantillonnage à partir d'une seule population source,

- la création de stocks composites à partir de plusieurs populations.

Enfin, nous examinerons dans une troisième partie les problèmes liés à la conservation de ces stocks en captivité pendant plusieurs générations.
\end{abstract}

\section{CAS D'UNE POPULATION SOURCE}

Dans un certain nombre de cas, on peut souhaiter ne constituer les stocks qu'à partir d'une seule population source. II peut s'agir par exemple de l'existence de garanties sanitaires particulières sur une population, de la volonté de sauvegarder une population donnée dans une optique de repeuplement (cas du saumon de l'Allier ou de l'esturgeon de la Gironde) ou du choix délibéré d'une population sur la base de performances qui se sont révélées satisfaisantes.

La question qui se pose alors est de collecter une fraction aussi large que possible de la variabilité génétique de la population. Empiriquement, il est clair que l'échantillon prélevé devra présenter un effectif important et ce problème est souvent considéré comme limitant, notamment lorsque la population source est difficilement accessible ou de faible effectif. Cependant, en termes génétique, la notion de taille de l'échantillon peut être notablement différente de celle de son effectif numérique et il importe de la préciser.

\section{DÉFINITION DE L'EFFECTIF GÉNÉTIQUE}

L'introduction se fait le plus souvent au stade d'œufs fécondés issus de quelques reproducteurs. Si $\mathrm{N}$ est l'effectif numérique de cet échantillon, on peut estimer la part de variance génétique de l'échantillon par rapport à celle de la population source par la formule (KIMURA et CROW, 1963; LACAVA et HUGHES, 1984) :

$$
\frac{V_{E}}{V_{T}}=1-\frac{1}{2 N_{\theta}}
$$

$N_{e}$ étant l'effectif génétique (ou effectif efficace) de l'échantillon. On l'obtient en calculant d'abord l'effectif efficace de reproducteur pour chaque sexe:

$$
N_{\theta m}=\frac{N_{m} K_{m}-i}{K_{m}-1+\frac{V_{m}}{K_{m}}} \quad \text { (2) } \quad N_{e}=\frac{N_{t} K_{t}-2}{K_{t}-1+\frac{V_{t}}{K_{t}}}
$$


- $N_{e m}$ et $N_{e f}$ sont les effectifs efficaces des reproducteurs mâles et femelles, $N_{m}$ et $N_{1}$ étant les nombres réels de reproducteurs mâles et femelles utilisés.

- $K_{m}$ et $K_{f}$ représentent la moyenne du nombre de descendants par reproducteur mâles et femelles. Ainsi, si l'échantillon comprend au total $\mathrm{N}$ individus, on a

$$
K_{m}=\frac{N}{N_{m}}
$$

$$
K_{f}=\frac{N}{N_{f}}
$$

$V_{m}$ et $V_{i}$ sont les variances de ces nombres de descendants entre reproducteurs mâles et femelles Enfin, l'effectif efficace de l'échantillon est obtenu par:

$$
\frac{4}{N_{e}}=\frac{1}{N_{e m}}+\frac{1}{N_{e 1}}
$$

En combinant les différentes relations, on peut écrire également :

$$
\frac{4}{N_{0}}=\frac{\left(K_{m}+\frac{V_{m}}{K_{m}}+\left(K_{f}+\frac{V_{f}}{K_{f}}\right)-2\right.}{N-2}
$$

\section{FACTEURS DE VARIATION}

Examinons plus précisément les conséquences de cette formule. Trois paramètres vont donc jouer un rôle important pour maximiser $N_{e}$ pour une valeur donnée de $N$ :

- le nombre de reproducteurs utilisés : pour un èchantillon introduit d'un effectif donné, ce nombre peut en effet varier dans des proportions considérables chez les espèces aquatiques à forte fertilité. Un seul couple peut être à l'origine d'un échantillon de plusieurs millions de juvéniles. Il convient donc d'accorder une grande importance à ce paramètre qui joue un rôle beaucoup plus capital que l'effectif numérique de l'échantillon introduit. Ainsi, en supposant que chaque reproducteur contribue de la même manière à l'échantillon, on a (voir plus loin) $\frac{V}{K}=1$, d'où :

$$
N_{\theta}=\frac{4(N-2)}{K_{m}+K_{f}}
$$

Pour un échantillon de 10.000 individus issus de 4 mâles et 4 femelles, on aura $K_{m}=K_{f}=2.500$ d'ou $N_{\epsilon} \simeq 8$ alors que pour un échantillon de seulement 100 individus issus de 5 mâles et 5 femelles, $K_{m}=K_{1}=20$, d'où $N_{e}=9,8$.

- la proportion des sexes dans le stock de reproducteurs utilisés : la figure 1 illustre cet aspect. II apparaît que si un nombre relativement faible de reproducteurs est suffisant lorsque le rapport des sexes est équilibré. l'existence d'un déséquilibre impose de recourir à un effectif beaucoup plus élevé. Ainsi la variabilité collectèe est plus faible avec 5 femelles et 95 mâles (soit 100 reproducteurs) qu'avec 10 femelles et 10 mâles, soit seulement 20 reproducteurs (97,2 contre $97,5 \%)$. Le plus souvent le nombre de femelles constitue effectivement un facteur limitant, du fait soit de la difficulté de capturer les animaux au bon moment, soit de la volonté de ne pas trop amoindrir le potentiel reproducteur de la population source. Cependant, la compensation par le recours à un nombre important de mâles n'est que partielle : la formule (6) montre en effet que quels que soient le nombre $\mathrm{N}$ mde mâles utilisés et la taille de l'échantillon, l'effectif génétique sera donc toujours inférieur à 4 fois le nombre de femelles utilisées. 


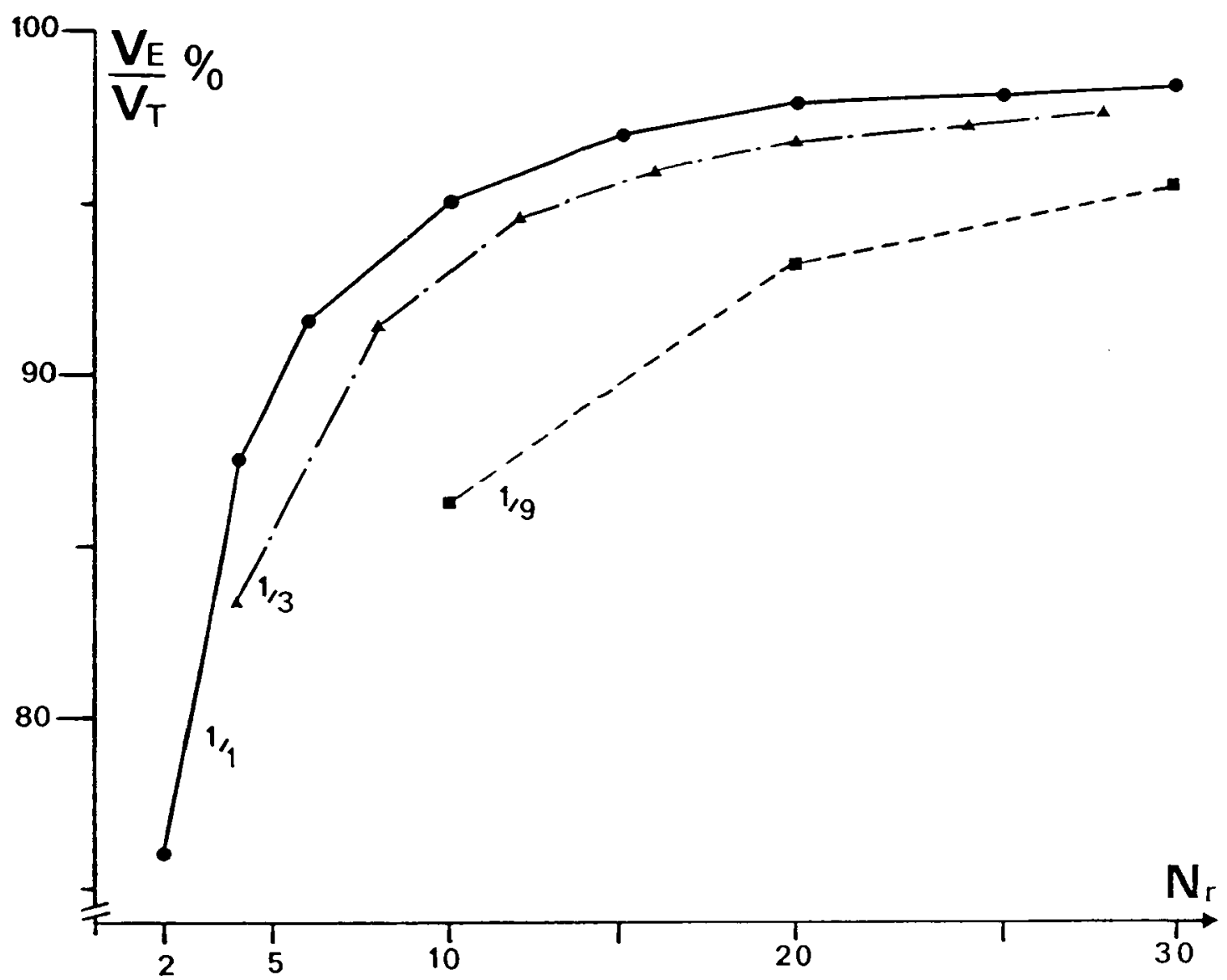

figure 1 : Part de la variance génétique (en $\%$ ) totale de la population introduite dans un échantillon issu de $N_{r}$ reproducteurs contenant une proportion variable de femelles. $1 / 1$ sex ratio équilibrée $1 \sigma^{7 / 1} / \mathrm{Q}$

$1 / 33$ mâles pour une femelle $1 / 99$ mâles pour une femelle

figure 1 : Part of the total genetic variance of the initial population (in \%) collected in a sample resulting from a group of $N_{\mathbf{r}}$ spawners with various sex ratios.

$1 / 1$ male for 1 female

$1 / 33$ males for 1 female

$1 / 99$ males for 1 female.

- la variance de la taille des familles, liée à la contribution de chaque reproducteur à la constitution de l'èchantillon de juvéniles. Dans le cas théorique où tous les reproducteurs apportent une contribution égale à la constitution de la nouvelle génération, on peut assimiler la constitution de l'échantillon à un tirage aléatoire décrit par une distribution dite "de Poisson", qui possède la propriété intéressante d'avoir une variance égale à son espérance. La formule (8) peut donc être utilisée. Cependant, cette hypothèse apparaît peu réaliste chez les espèces aquatiques. En particulier, la qualité des ovules collectés peut être inégale d'une femelle à l'autre et les taux de fécondation et d'éclosion peuvent varier dans des proportions considérables. De ce fait, les $\checkmark$ contributions de chaque femelle à l'échantillon sont différentes et le rapport $\frac{\mathrm{K}}{\mathrm{K}}$ peut alors être très supérieur à 1, d'où une éduction marquée de l'effectif efficace femelle (tableau I, cas C). Pour minimiser ce phénomène, il est donc souhaitable de conserver séparément les différentes pontes pendant toute la période où des variations importantes de survie liées à la femelle peuvent se manifester (développement embryonnaire, éclosion et résorption vésiculaire et si possible début de l'alimentation exogène). A l'issue de cette phase, on pourra regrouper les lots en prélevant dans chacun d'entre eux : 
Tableau I : Effet de la variance de la taille des familles sur l'effectif génétique.

L'effectif efficace étant donné par $N_{0}=\frac{N-2}{K-1+\frac{V}{K}}$

le cas $A$ représente une distribution aléatoire issue d'un tirage où les femelles sont équireprésentées. L'effectif efficace est peu intérieur à un cas d'égalité parfaite (B). Le cas C illustre l'effet d'une participation très inégale des femelles. L'effectif efficace est deux fois plus faible alors que l'effectif $\mathbf{N}$ est plus important. Les cas D à $\mathbf{F}$ illustrent deux stratégies de troncature appliquée au cas $\mathbf{C}$ (voir figure 2 ).

Table I : Effect of the variance of family size on the value of the effective number

(see the text)

$$
N_{0}=\frac{N-2}{K-1+\frac{V}{K}}
$$

$A$ is the case of a random distribution with the same probability of occurence for each female,

$B$ the effective number is slightly higher in the case of perfect equality of family size,

C when the occurrence of the females are very unequal, the effective size can be highly reduced,

D, E, F, see figure 2.

\begin{tabular}{|c|cccccccccc|ccc|}
\hline$q$ & 1 & 2 & 3 & 4 & 5 & 6 & 7 & 8 & 9 & 10 & $\mathrm{~N}$ & $\frac{\mathrm{V}}{\mathrm{K}}$ & $\mathrm{N}_{\mathrm{e}}$ \\
\hline $\mathrm{A}$ & 9 & 5 & 4 & 7 & 6 & 3 & 1 & 4 & 5 & 6 & 50 & 0,88 & 9,83 \\
$\mathrm{~B}$ & 5 & 5 & 5 & 5 & 5 & 5 & 5 & 5 & 5 & 5 & 50 & 0 & 12 \\
$\mathrm{C}$ & 27 & 25 & 10 & 8 & 4 & 2 & 1 & 1 & 1 & 1 & 80 & 11,3 & 4,37 \\
$\mathrm{D}$ & 27 & 25 & 10 & 8 & 4 & 2 & - & - & - & - & 76 & 7,57 & 3,95 \\
$\mathrm{E}$ & 12 & 10 & 10 & 8 & 4 & 2 & 1 & 1 & 1 & 1 & 50 & 3,64 & 6,28 \\
$\mathrm{~F}$ & 8 & 8 & 8 & 8 & 4 & 2 & 1 & 1 & 1 & 1 & 42 & 2,47 & 7,05 \\
\hline
\end{tabular}

- des effectifs égaux si les pontes ont été fécondées chacune par un même nombre de mâles,

- des effectifs proportionnels à leur effectif génétique si les pontes ont été fécondées par des mélanges de spermes issus de mâles différents*. Cette pratique peut apparaître paradoxale car elle conduit à ajuster les prélèvements sur le lot ayant présenté la plus faible survie au lieu de regrouper tous les survivants des différentes pontes. Cette stratégie de troncature par le haut (tableau I, E et F) est cependant à recommander, l'effectif efficace étant beaucoup plus sensible à la variance de la taille de famille qu'à leur effectif moyen (figure 2).

D'autre part, il peut s'avérer utile de répartir dans le temps l'effort d'échantillonnage. Cela peut permettre d'une part de collecter un effectif génétique plus important, notamment lorsque le nombre de femelles constitue un facteur limitant, d'autre part de tenir compte d'éventuelles " hétérogénéités" de la population de reproducteurs. L'ensemble des reproducteurs présents au même endroit ou matures au même moment peut en effet, même si son effectif est important, ne représenter qu'une fraction de la variabilité totale, notamment dans les populations de migrateurs présentant un "homing" (retour au lieu de naissance) précis. L'échantillonnage gagnera donc à être rèpartisur la période de reproduction ou répété pendant plusieurs années consécutives ou non.

\footnotetext{
" ainsi, si l'on dispose de trois pontes A, B, C fécondées respectivement par 5, 10 et 20 mâles, les effectifs génétiques de ces pontes $\left(\mathrm{Ne}=\frac{4 \mathrm{Nm}}{\mathrm{Nm}+1}\right)$ sont respectivement de 3,20;3,64 et 3,81. On regroupera donc par
} exemple 320 alevins issus de la ponte A, 364 issus de la ponte $B$ et 381 issus de la ponte C. 


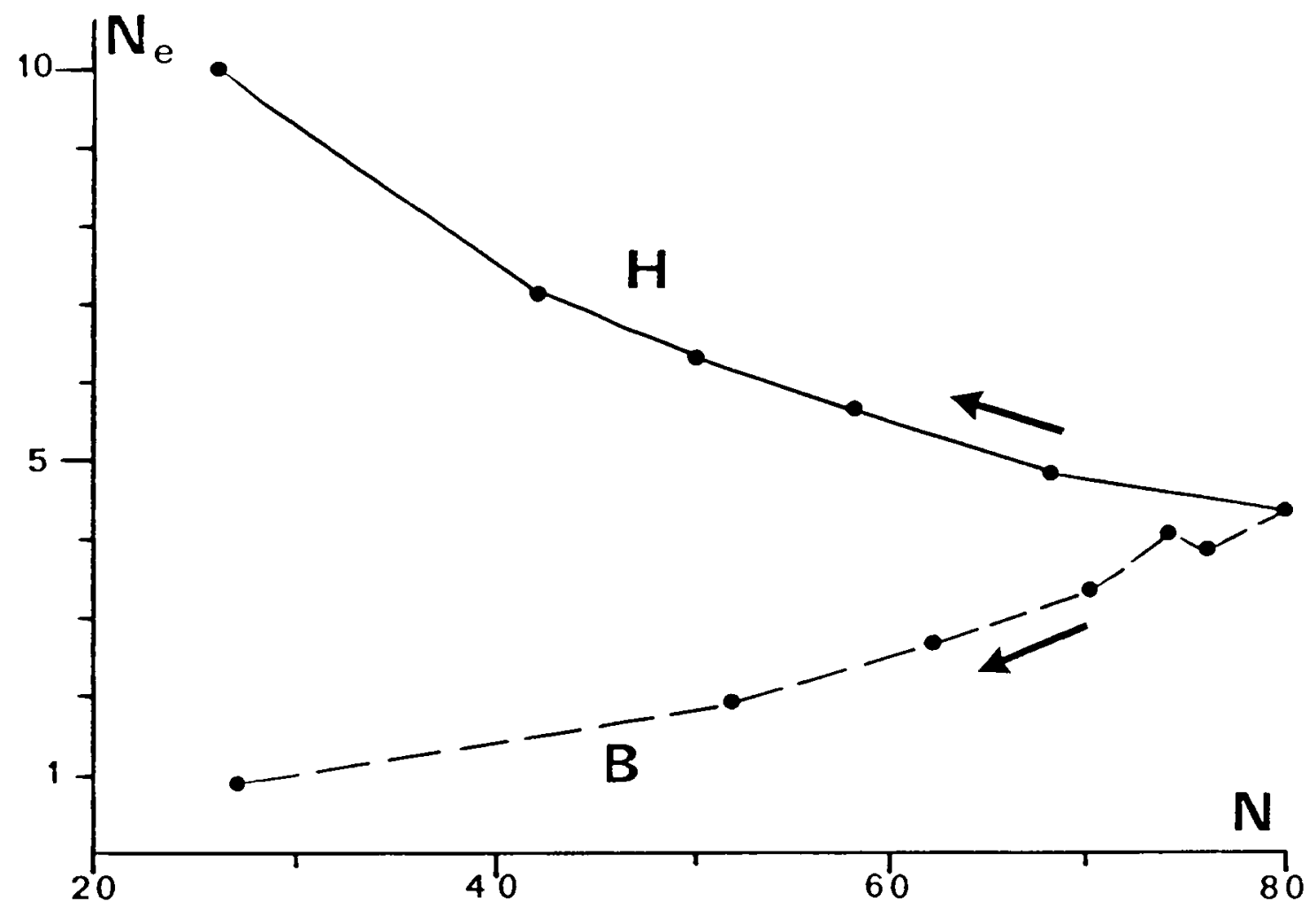

figure 2 : Exemple d'évolution de l'effectif génétique $N_{e}$ en fonction de deux stratégies d'égalisation de la taille des familles.

H égalisation par le haut (réduction de l'effectif des familles les plus nombreuses, cas E et F)

B égalisation par le bas (élimination des familles les moins nombreuses, cas D) Les données de départ correspondent au cas $\mathbf{C}$ du tableau $\mathbf{I}$.

figure 2 : Effect of two alternative strategies for equalizing family size. The initia! situation is case $\mathbf{C}$ (Table I).

$H$ rediction of the size of the more numerous families (cases $E$ and $F$ ). The effective size $\mathrm{N}_{\mathrm{e}}$ increases.

$\mathrm{B}$ elimination of the less numerous families (case D). The effective size $\mathrm{Ne}_{\mathrm{e}}$ decreases. 


\section{CONCLUSION}

Si l'on revient à la formule (1), il est clair qu'en respectant les précautions définies précédemment, il est relativement aisé de collecter une fraction importante de la variabilité génétique de la population source (figure 3) : si l'on fixe comme objectif "standard" d'introduire $95 \%$ de la variabilité initiale, ce but est par exemple atteint avec un échantillon de 100 individus issus de 3 femelles fécondées chacune par 5 mâles différents. Avec 15 femelles également fécondées par 5 mâles, la perte de variabilité n'est que de $1 \%$. Ces principes devraient donc être largement diffusés auprès des gestionnaires d'écloserie.
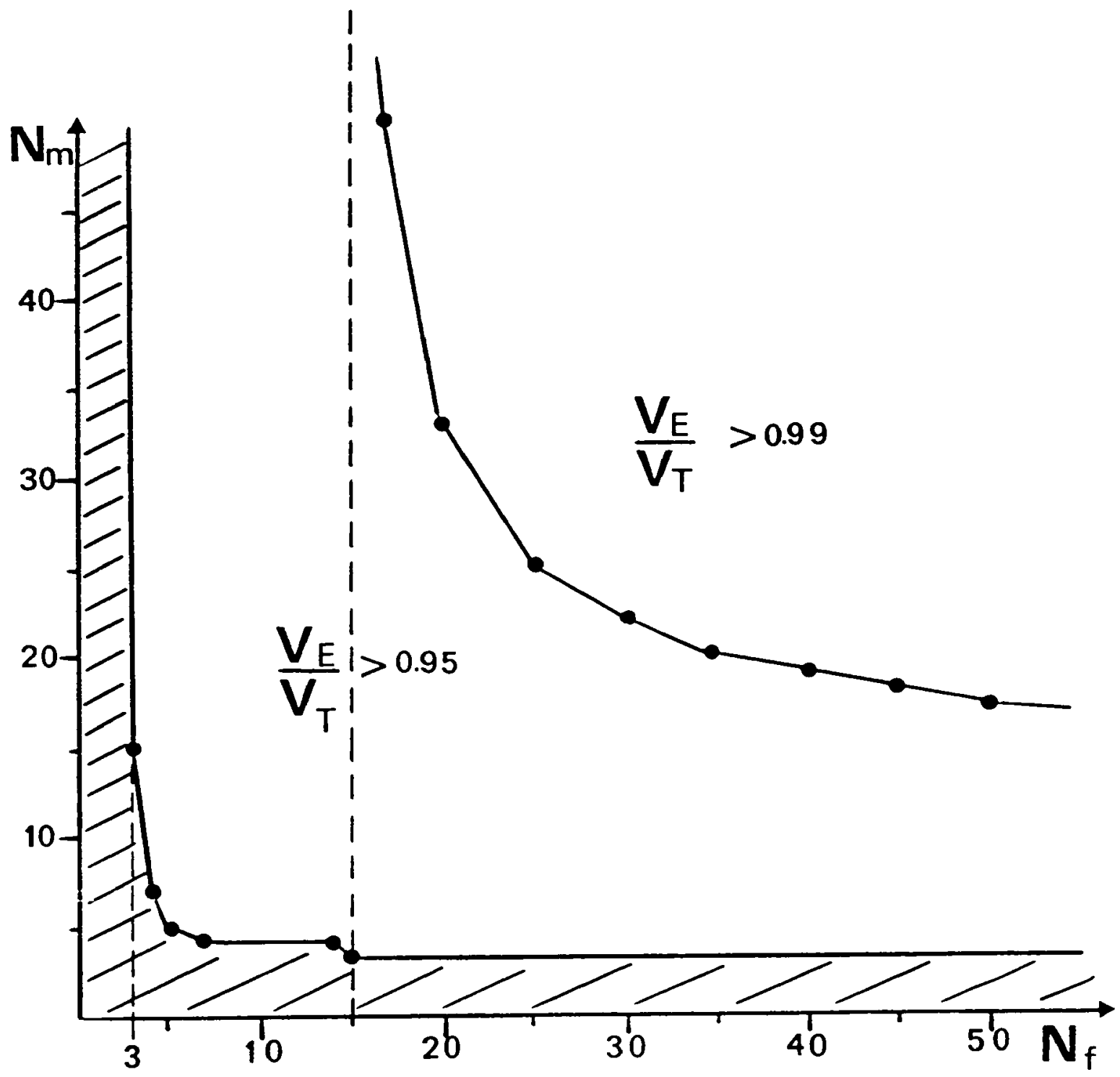

figure 3 : Nombre de reproducteurs mâles $\left(N_{m}\right)$ et femelles $\left(N_{f}\right)$ nécessaires pour introduire respectivement $95 \%$ et $99 \%$ de la variabilité génétique totale de la population. Les nombres minimums de femelles nécessaires dans ces deux cas sont respectivement de 3 et 15 pour un nombre de mâles supérieur à 50.

figure 3 : Number of male $\left(N_{m}\right)$ and female $\left(N_{f}\right)$ spawners required to collect at least 95 or $99 \%$ of the total genetic variance of the source population : for a number of males larger than 50, the minimal numbers of females are respectively 3 and 15. 


\section{CAS DE PLUSIEURS POPULATIONS-SOURCES}

Il est fréquent que plusieurs populations, naturelles ou d'élevage, puissent être identifiées au sein de l'espèce et la stratégie à adopter pour créer un stock de reproducteurs devient plus complexe. Faut-il ne choisir qu'une population (et dans ce cas laquelle?) ou au contraire réaliser une population "synthétique" en mélangeant plusieurs de ces populations? Dans la plupart des cas les informations disponibles sur ces populations sont limitées à des données historiques souvent imprécises (portant sur l'origine des populations d'élevage, les échanges éventuels entre populations) ou à des observations biologiques in situ (croissance, morphologie, période de reproduction) dont l'interprétation en termes de différenciation génétique est problématique. II est donc dans ce cas nécessaire de préciser l'organisation de la variabilité génétique intra et interpopulations pour définir la stratégie d'èchantillonnage.

\section{LES MÉTHODES DE DESCRIPTION}

Les méthodes de description de la variabilité génétique ont été décrites précédemment. Rappelons qu'elles peuvent concerner:

- les caractères "quantitatifs" directement observables comme la pigmentation, la morphologie, la fécondité... Dans ce cas, il est nécessaire de pouvoir comparer les populations après introduction dans le même milieu, opération longue, coûteuse et ne fournissant qu'une réponse partielle à la question du rôle des facteurs génétiques sur la différenciation observée in situ (voir l'article de F. KRIEG dans ce numéro). Nous rappellerons seulement ici que les principes définis au paragraphe précédent s'appliquent même au cas d'une introduction transitoire d'une population à des fins de testage. En effet, l'échantillon doit présenter une variabilité suffisante pour être représentatif de la population. On montre en effet que pour un échantillon de taille $N$ et d'effectif génétique $\mathrm{N}_{\mathrm{e}}$ la précision o (en \%) obtenue sur la valeur génétique de la population d'origine vaut :

$$
\sigma=C V \sqrt{\frac{h^{2}}{N_{e}}+\frac{2-h^{2}}{2 N}}
$$

C V est le coefficient de variation observé du caractère considéré, $h^{2}$ est l'héritabilité de ce caractère (proportion de la variance observée liée à la variation génétique entre individus).

Ainsi, pour un caractère comme la performance de croissance, présentant un coefficient de variation de l'ordre de $30 \%$ et avec une valeur d'héritabilité de 0,2 , il faudra au moins 100 individus issus de 5 mâles et 5 femelles pour estimer la valeur génétique avec une précision de $5 \%$, ce qui est faible. Pour obtenir une précision supérieure, l'effectif efficace $\mathrm{Ne}$ constituera généralement le facteur limitant. Ainsi, il faudra au moins atteindre $\mathrm{Ne}_{\mathrm{e}}=60$ (30 mâles et 30 femelles par exemple) pour que la précision atteigne $2 \%$ même en mesurant 1.000 individus.

- des caractères non sensibles à l'action directe du milieu comme ceux obtenus par les études caryologiques ou moléculaires, décrits dans les articles précédents. Rappelons seulement que ces études fournissent trois types de paramètres globaux:

- La variation génétique intrapopulation, mesurée par la fréquence moyenne d'individus hétérozygotes pour l'ensemble des gènes étudiés;

- La différenciation entre les populations, qui permet de mettre en évidence les populations voisines et les grands groupes biogéographiques;

- Le taux de différenciation de l'espèce, obtenu en combinant les deux informations précédentes (Rapport de la variation génétique moyenne inter-populations à la variation génétique totale).

\section{LES RÉSULTATS}

L'article de R. GUYOMARD fournit un exemple détaillé des résultats ontenus chez la truite fario. En combinant ces résultats à des études conduites chez d'autres salmonidés (STAHL, 1981 et GUYOMARD, $1987 \mathrm{chez}$ le saumon atlantique; ALLENDORF et LEARY, $1988 \mathrm{chez}$ la truite gorge coupée; MINDAR et al., $1988 \mathrm{chez}$ l'omble arctique), il est possible d'avancer plusieurs conclusions de portée générale:

- la variabilité au sein d'une population naturelle donnée dépasse le plus souvent $50 \%$ de la variabilité totale de l'espèce. Sans discuter ici des mécanismes évolutifs permettant d'expliquer un tel résultat, il convient donc d'abandonner définitivement une conception selon laquelle une population locale serait constituée d'individus tous identiques entre eux et différents de ceux 
d'une autre population. Cette notion de "pureté génétique" d"une race locale doit être remplacée par une vision selon laquelle une population naturelle peut présenter certes une certaine originalité génétique moyenne mais constitue avant tout, sauf exception, un ensemble polymorphe possédant une réserve importante de variabilité génétique.

- la variabilité au sein des stocks domestiques n'est pas systématiquement inférieure à celle observée chez les populations naturelles (tableau II). Si des cas de réduction marquée de variabilité peuvent être observés, certains stocks peuvent également présenter une variabilité élevée, liée éventuellement à leur origine composite.

Tableau II : Comparaison de l'hétérozygotie intrapopulation dans des populations sauvages et domestiques de salmonidés. Le tableau donne la valeur moyenne de l'hétérozygotie $(H)$, le nombre et l'origine des populations et les valeurs extrêmes d'hétérozygotie observées dans ces populations. (R)

* I'hétérozygotie est calculée sur un échantillon de locus polymorphes et est donc surestimée.

Table II : Comparison of intrapopulation heterozygosity in wild and domestic populations of salmonids. The table gives the mean value of heterozygosity $(H)$, the number and origin of the populations and the extremes values observed in those populations. * heterozygosity is estimated with a set of polymorphic loci and thus overestimated.

\begin{tabular}{|c|c|c|c|c|}
\hline Species & & wild populations & domestic populations & References \\
\hline Salmo clarkf1 & $\overline{\mathbf{u}}$ & $2,2 \quad(1$, USA $)$ & $2,7 \quad(1, U S A)$ & ALLENDORF and LEARY, 1988 \\
\hline Salmo gairdneri & $\begin{array}{l}\overline{\mathbf{H}} \\
\mathrm{R} \\
\overline{\mathrm{H}} \\
\mathrm{R}\end{array}$ & $31,0 \quad(1$, USA $)$ & $\begin{array}{l}18,0 \quad(9, \text { USA }) \\
(8,6-29,5) \\
31,2 \quad(6, \text { FRANCE }) \\
(24,1-34,8)\end{array}$ & in GUYOMARD, $1981^{\star}$ \\
\hline Salmo salar & $\begin{array}{l}\overline{\mathrm{H}} \\
\mathrm{R} \\
\overrightarrow{\mathrm{H}}\end{array}$ & $\begin{array}{c}2,8 \quad(9, \text { SWEDEN }) \\
(2,2-3,5) \\
21,8 \quad(1, \text { IRELAND })\end{array}$ & $\begin{aligned} 2,2 & (9, \text { SWEDEN }) \\
(1,5 & -3,1) \\
17,3 & (1, \text { IRELAND })\end{aligned}$ & $\begin{array}{l}\text { STÅHL, } 1983 \\
\text { CROSS and KING, } 1983^{\star}\end{array}$ \\
\hline Salmo trutta & $\begin{array}{l}\overrightarrow{\mathrm{H}} \\
\mathrm{R} \\
\mathrm{R} \\
\mathrm{R}\end{array}$ & $\begin{array}{c}6,79(19, \text { WESï } \\
\text { EUROPE }) \\
(1,8-10,8) \\
4,41 \quad(13, \text { SOUTH } \\
\text { FRANCF }) \\
(0,0-8,2)\end{array}$ & $7,94 \quad(5$, FRANCE) & NIYYMARD, 1989 \\
\hline
\end{tabular}

- les différenciations génétiques observées entre les populations ne confirment pas toujours des hypothèses basées sur des observations écoéthologiques. Ainsi, or accorde souvent chez les salmonidés une importance majeure au caractère migrateur ou sédentaire des populations, voire à leur mode de vie (populations lacustres ou de rivière), au point parfois de considérer cc.mme des sous-espèces ces différents écotypes. ¿̇ cas de l'omble arctique (salvelinus alpinus) démontre clairement (MINDAR et al., 1988) que ces différenciations écologiques apparaissent génétiquement minimes par rapport à la différenciation géographique entre populations de même écotype. Inverzement, le cas de la truite fardée (salmo clarkii) illustre la nécessité de gérer parfois comme des sous-espèces différentes des populations écologiquement proches (ALLENDORF et LEARY, 1988). Les résultats obtenus sur la truite fario apportent des conclusions tout-à-fait similaires.

- la structure génétique de l'espèce (taux de différenciation) peut varier de manière importante même entre espèces proches et nécessite donc à chaque fois une étude particulière. Ainsi, parmi 54 populations d'un saumon cu Pacifique (Oncorhynchus keta), le taux de différenciation n'est que de $2 \%$ (98\% cie variation intrapopulation). Ce taux atteint $\mathrm{f}$. 
truite fardée (Salmo clarkii) alors qu'il est seulement de $16 \%$ dans une espèce très voisine, la truite arc-en-ciel (Salmo gairdneri) (GUYOMARD, 1989).

\section{INTERPRÉTATION ET APPLICATIONS}

Comment prendre en compte ces données pour la constitution d'un stock de reproducteurs? De manière générale, l'intérêt de constituer un stock présentant au départ une large variabilité génétique se justifie par deux types d'arguments :

- A court terme, il peut exister une certaine relation entre la variabilité génétique d'un stock et ses performances en élevage. Quoique non systématiques, de telles relations ont été mises en évidence à plusieurs reprises chez des espèces aquacoles et nous en donnerons quelques exemples. Ainsi, une étude de la durée du développement embryonnaire dans 6 populations naturelles de truite arc-en-ciel (salmo gairdneri) conduite par FERGUSSON et al. (1985) met en évidence une relation positive entre l'hétérozygotie moyenne de la souche et sa rapidité de développement (figure 4). L'écart est d'environ 4 jours (10\%) lorsque les niveaux d'hétérozygotie passent de 4 à $8 \%$. Ces variations se retrouvent également chez cette espèce lorsque l'on étudie au sein d'une population les relations entre l'hétérozygotie individuelle, mesurée par les marqueurs enzymatiques, et la précocité d'éclosion (DANZMANN et al., 1986). De même, chez les mollusques, des relations nettes ont été mises en évidence à plusieurs reprises entre la vitesse de croissance et l'hétérozygotie individuelle (ZOUROS et FOLTZ, 1987 pour une revue). La figure 5 donne un exemple de ce phénomène.

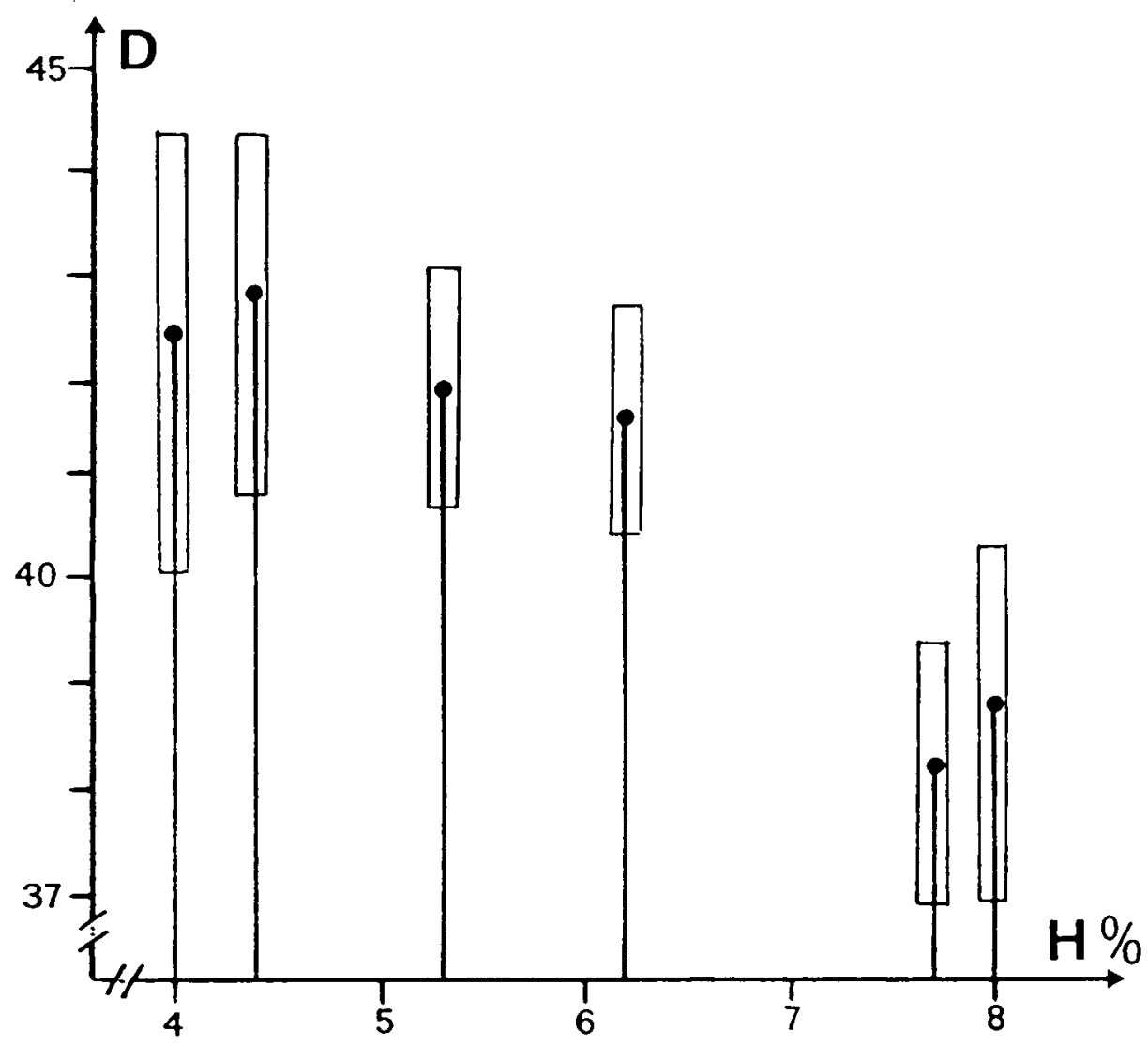

figure 4 : Relation entre la durée du développement embryonnaire D (en jours) et l'hétérozygotie moyenne $H$ dans six populations de truite arc-en-ciel Salmo gairdneri. Le rectangle indique l'écart-type (d’après FERGUSON et al., 1985).

figure 4 : Relationship between the duration of embryonic development $D$ (in days) and the mean heterozygosity $H$ in six populations of rainbow trout Salmo gairdneri. The rectangle gives the standard deviation around the mean (from FERGUSON et al., 1985). 

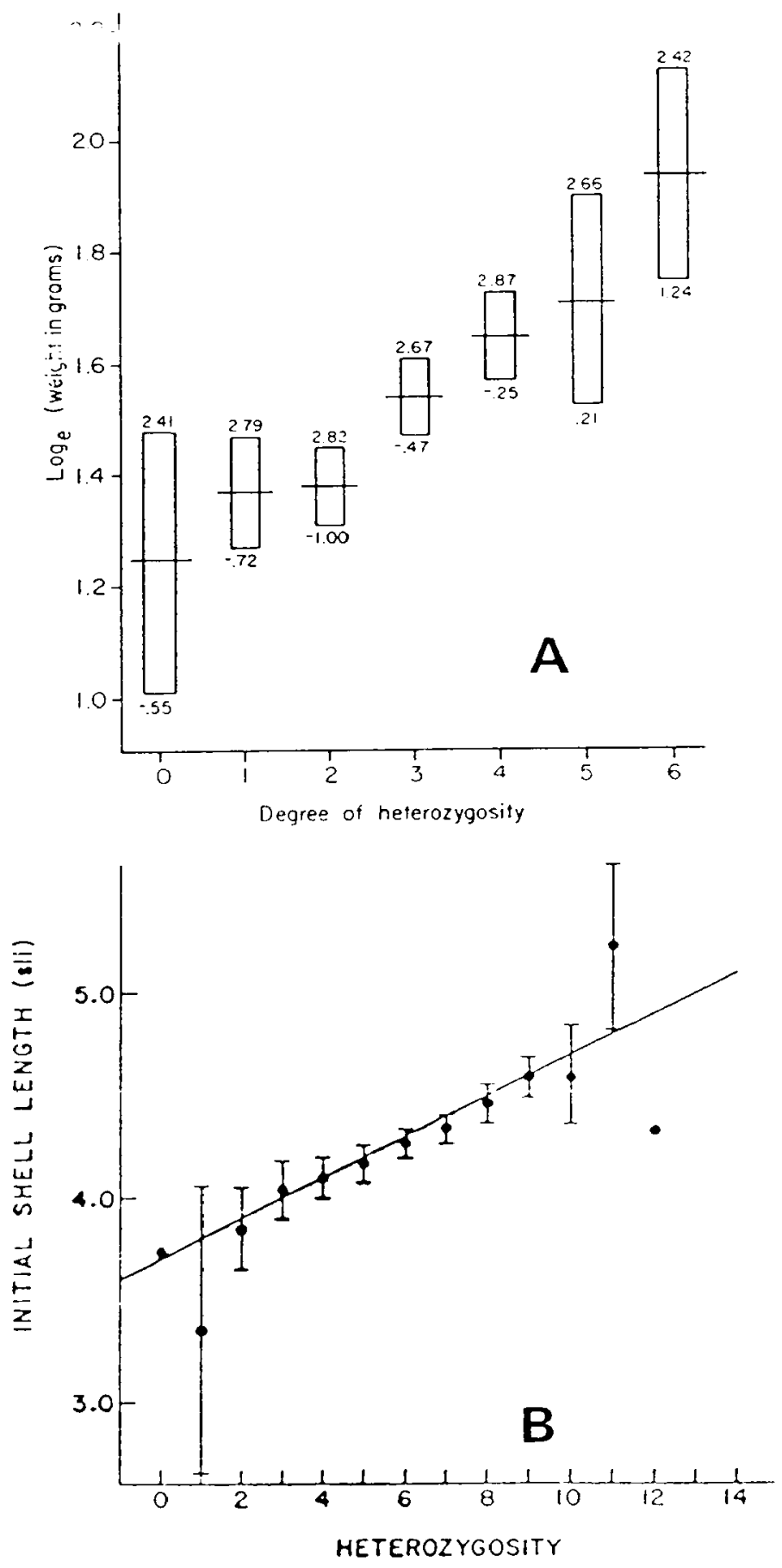

figure 5 : Deux exemples de relations positives entre l'hétérozygotie (estimée par le nombre de systèmes enzymatiques à l'état hétérozygote dans un individu donné) et le potentiel de croissance chez les mollusques.

A poids des individus à 1 an chez l'huitre américaine Crassostrea virginica (FOLTZ et al., 1983)

B longueur de la coquille à la fixation chez Mulinia lateralis (KOEHN et al., 1988)

figure 5 : Two examples of positive relationships between individual heterozygosity (estimated by the number of loci which are heterozygous in a given individual) and growth potential in Molluscs.

A weight of individuals at 1 year of age in the american oyster Crassostrea virginica (FOLTZ et al., 1983)

B length of the shell at settlement in Mulinia lateralis (KOEHN ot al., 1988). 
L'effet de l'hétérozygotie peut se révéler également non pas sur la valeur moyenne du caractère mais sur sa variabilité. Une meilleure "stabilité de développement" (developmental stability ou genetic homeostasis de LERNER, 1954) se traduirait chez les hétérozygotes par une plus faible variance des caractères. L'étude de FERGUSSON et al. (1985) déjà citée (figure 4) montre notamment un plus faible étalement de la période d'éclosion chez les populations les plus hétérozygotes. De même MITTON (1978) a pu démontrer chez un poecilidae, fundulus heteroclitus, que la variance interindividuelle des caractères méristiques (nombre d'écailles, nombre de rayons des nageoires) était généralement plus faible chez les individus hétérozygotes. Cette notion a été particulièrement développée chez les vertébrés par les études portant sur la dissymétrie bilatérale de ces caractères : à partir du dénombrement de caractères externes pairs (nombre de rayons des nageoires, de branchiospines, d'écailles sur la ligne latérale), on peut caractériser chaque individu ou chaque population par un indice de dissymétrie supposé mesurer sa "stabilité de développement" et relier cet indice aux mesures biochimiques d'hétérozygotie. Plusieurs exemples (figure 6) illustrent la pertinence de cette démarche en mettant en évidence une augmentation de l'indice de dissymètrie quant l'hétérozygotie diminue (LEARY et al., 1983), 1984, 1985a).
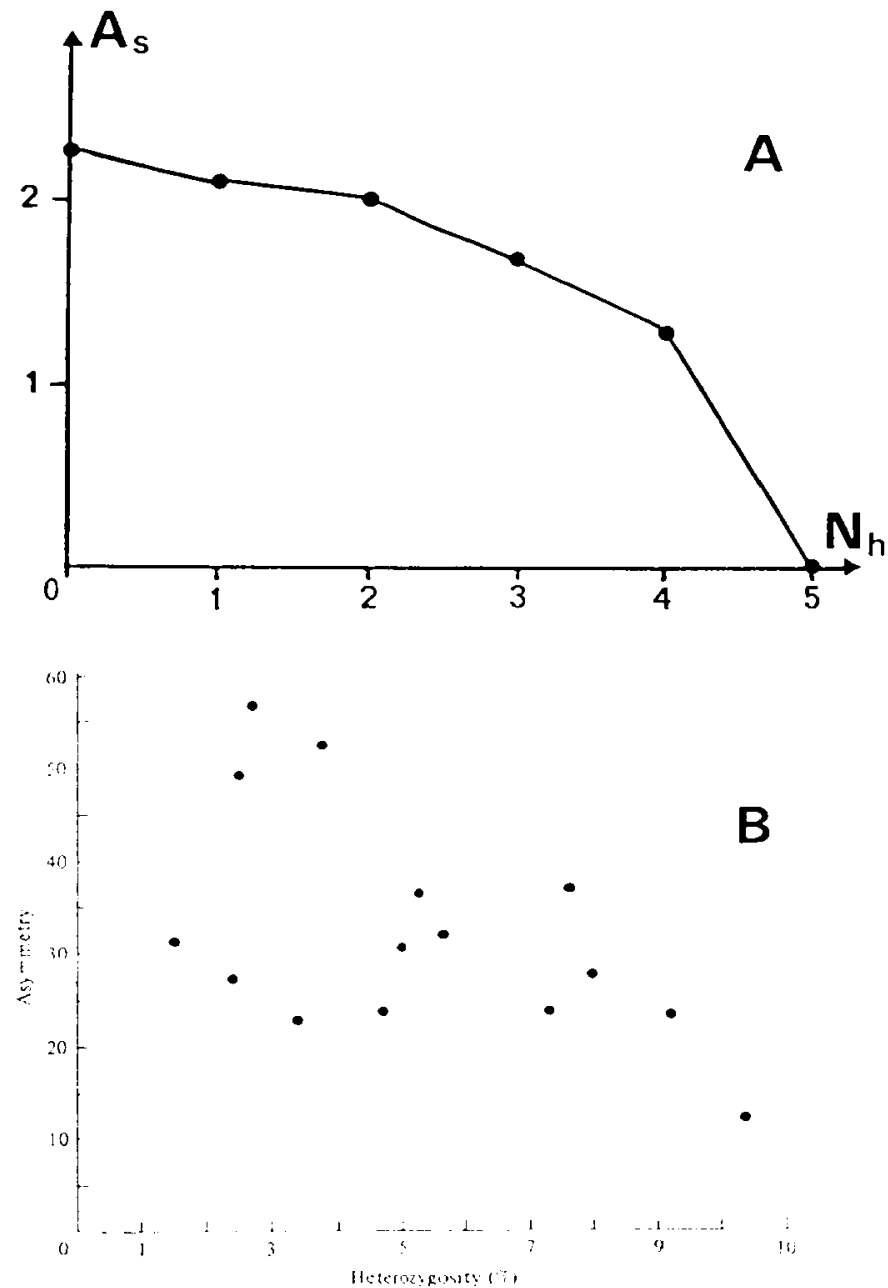

figure $6 \mathrm{~A}$ : Relation entre le nombre de locus à l'état hétérozygote $\mathbf{N}_{\mathrm{h}}$ et le nombre moyen de caractères présentant une dissymètrie gauche-droite $\left(A_{S}\right)$ dans une population de truite arc-en-ciel Salmo gairdneri (d'après LEARY et al., 1983).

figure 6 A : Individual relationship between number of heterozygous loci Nh and mean number of assymetric characters As in a rainbow trout population (from LEARY et al., 1983)

figure $6 \mathrm{~B} \quad$ : Relation entre l'hétérozygotie moyenne de la population $\overline{\mathrm{H}}$ et l'assymétrie bilatérale chez 14 populations de lézard Uta stansburiana (d'après SOULE, 1979).

figure 6 B : Relationship between mean heterozygosity and a symetry coefficient among 14 populations of the lizard Uta stansburiana (from SOULE, 1979). 

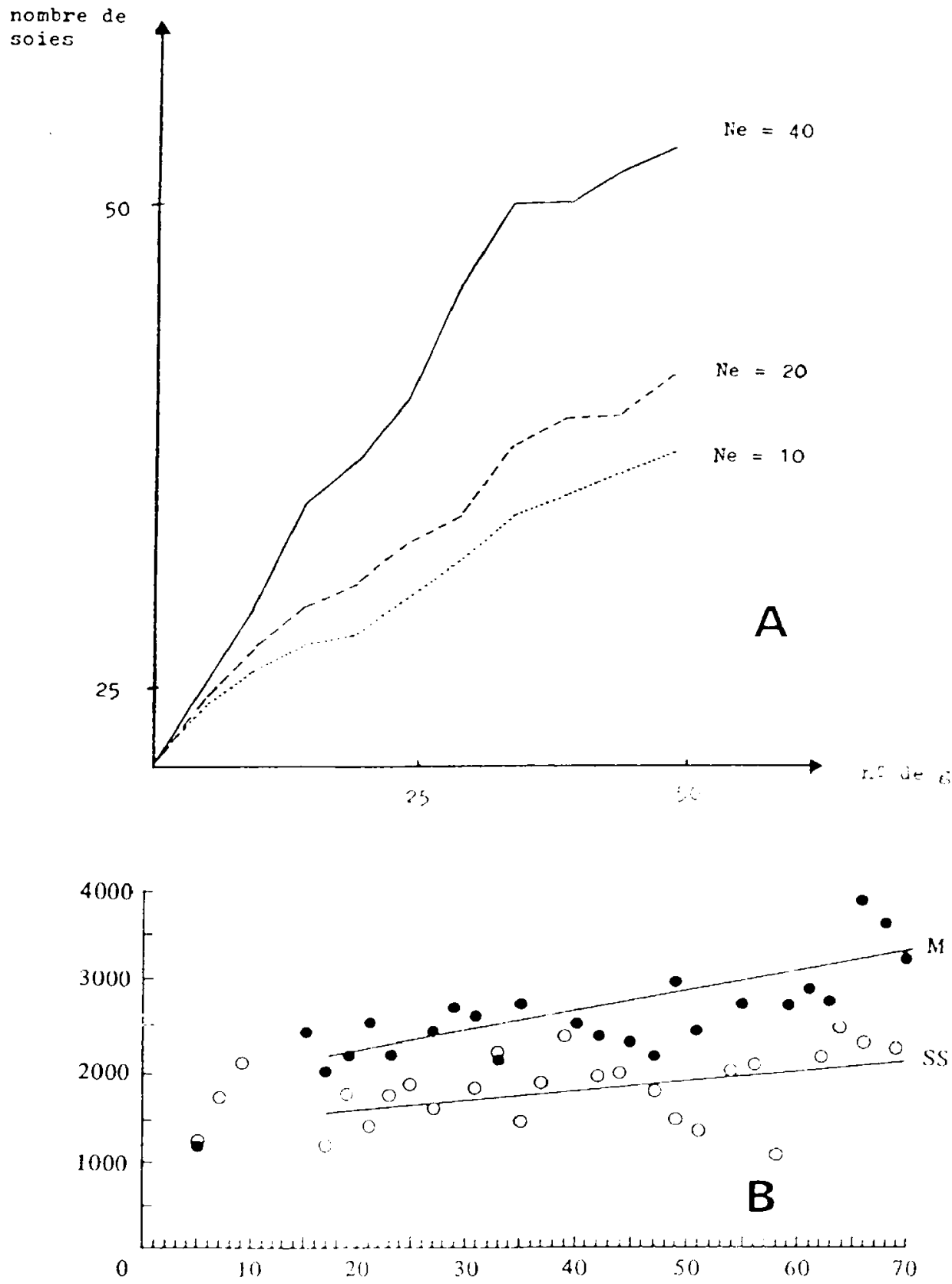

figure 7 : Deux exemples de relations entre la variabilité génétique et les capacités évolutives chez les drosophiles.

A Réponse à long terme à une sélection pour l'augmentation du nombre de soies sternopleurales en fonction de l'effectif efficace (JONES et al., 1969)

B Evolution démographique de deux populations expérimentales. SS est une population pure, $M$ une population issue du mélange de plusieurs lignées. Le taux d'accroissement est deux fois plus élevé pour M (AYALA, 1965).

figure 7 : Two examples of relationship between genetic variability and "evolutive capacity" in drosophilae.

A long term response to selection for the number of sternopleural bristles. $\mathrm{N}_{e}$ is the effective size of the selected population at each generation

B Demographic evolution of two experimental populations : SS is a single strain population, $\mathrm{M}$ a "synthetic" strain resulting from a mixing of different strains. The average rate of increase in population size is about twice larger in the $M$ population (from AYALA, 1965 in Frankel and Soule, 1981). 
Il va de soi que de telles approches ne peuvent prétendre remplacer une évaluation directe des performances des individus ou des populations: ces relations ne sont pas systématiques (voir notamment Mc ANDREW et al. 1986) chez la plie pleuronectes platessa. BEACHAM et WITHLER (1985) chez le saumon rose Oncorhynchus gorbuscha pour des résultats négatifs), ne concernent que certains caractères et les corrélations observées entre performance et hétérozygotie, lorsqu'elles sont significatives, n'expliquent généralement qu'une faible partie (10 à $30 \%$ ) de la variabilité totale du caractère. II n'est donc pas question de remplacer une amélioration directe des caractères par des méthodes visant à maximiser l'hétérozygotie. Cependant, ces illustrations nous semblent suffisantes pour justifier le choix a priori de populations présentant une hétézozygotie èlevée.

- A plus long terme, la capacité de réponse d'une population à un changement d'environnement est classiquement reliée à son niveau de variabilité génétique. Or la domestication, qu'elle s'accompagne ou non d'une sélection complémentaire pour certains caractères, constitue une "épreuve adaptative" importante dont le résultat final peut dépendre en partie de la diversité génétique initiale de la population. La réalisation expérimentale de telles études n'est guère envisageable dans le cas des especes aquacoles mais la figure 7 fournit un exemple de telles évolutions chez la drosophile.

Dans ces conditions, la constitution de stocks à partir du regroupement d'un grand nombre de populations naturelles pourrait apparaître comme une solution à préconiser systématiquement. Cependant, il nous semble nécessaire de moduler notablement cette proposition. En effet

- Dans le cas d'une espèce présentant un faible taux de différenciation, le choix d'une population présentant une forte hétérozygotie permet déjà de collecter une fraction importante de la variabilité totale. A la limite, lorsque l'hétérozygotie varie de manière importante entre les populations, on peut même montrer que le choix de la population ayant le plus fort taux d'hétérozygotie conduit à disposer d'une variabilité génétique plus large que celle d'une population synthétique regroupant systématiquement toutes les populations disponibles. On peut donc se contenter du regroupement des deux ou trois populations les plus hétérozygotes, ce qui permet en outre d'alléger l'effort de collecte. Le regroupement d'animaux vivants est en effet souvent nettement plus problématique que les prélèvements d'échantillons destinés à l'analyse biochimique. Dans cette démarche un testage des performances des quelques populations intéressantes peut constituer un critère de choix complémentaire.

- Dans le cas d'espèces présentant un fort taux de différenciation, la réalisation de populations synthétiques peut conduire à des conséquences négatives à plus ou moins long terme qu'il convient d'évaluer. Auparavant, un commentaire est sans doute nécessaire sur la notion de fort taux de différenciation. En effet, un modèle simple connu sous le titre d" horloge moléculaire" (NEI, 1975) permet de relier la distance génétique entre deux populations et l'ancienneté de leur différenciation. Ainsi dans l'exemple de la figure $8 \mathrm{~A}$, tirèe d'une comparaison de cinq populations de saumon atlantique suédoises, les distances génétiques moyennes entre populations sont de l'ordre de $0.3 \%$ et correspondraient à un isolement d'environ 15.000 ans. On pourrait donc être tenté d'adopter une attitude de grande prudence vis-à-vis du bouleversement éventuel créé par le regroupement de telles populations. Cependant, plusieurs exemples conduisent à s'interroger sur la validité de cette approche. En particulier les expériences d'introduction de salmonidés aux îles Kerguelen, conduites depuis une quarantaine d'années ont donné lieu à partir d'une même population initiale à des stocks présentant actuellement des niveaux de différenciation du même ordre (figure 8B: GUYOMARD, 1984). II semble donc difficile d'accorder une signification évolutive majeure à des niveaux de différenciation de l'ordre de 1 à $2 \%$, à moins qu'ils ne soient confortés par l'existence, pour certains systèmes enzymatiques, d'allèles marqueurs spécifiques de certaines populations.

Ainsi dans une étude sur deux populations de truite fario coexistant dans un même lac et présentant une distance génétique relativement faible $(2,5 \%)$. ALLENDORF et al. (1976) ont pu montrer que chaque population possédait pour un système enzymatique (LDH 1) un allèle spécifique (commun à tous les individus d'une population et absent dans l'autre) qui attestait l'absence de reproduction croisée entre ces deux populations. Un tel phénomène, s'il doit être pris en compte dans une optique de gestion des populations naturelles, ne doit pas être interprété comme une incompatibilité génétique entre les deux stocks. L'isolement reproducteur peut résulter de phénomènes éco-éthologiques variés (décalage des périodes ou des zones de ponte par exemple) et seule une étude expérimentale permettrait de conclure à la possibilité ou non d'obtenir une descendance viable en croisant ces populations.

Pour des taux de différenciation supérieurs (5 à $10 \%$ ). les conséquences éventuelles du mélange de populations sont de deux ordres

- Les individus de première génération issus du croisement entre les populations, peuvent présenter une viabilité réduite. Ce phenomène est connu dans le cas des hybrides interspécifiques (voir notamment CHEVASSUS, 1983). Par contre, les exemples dans le cas de croise- 


\section{GENETIC DISTANCE}
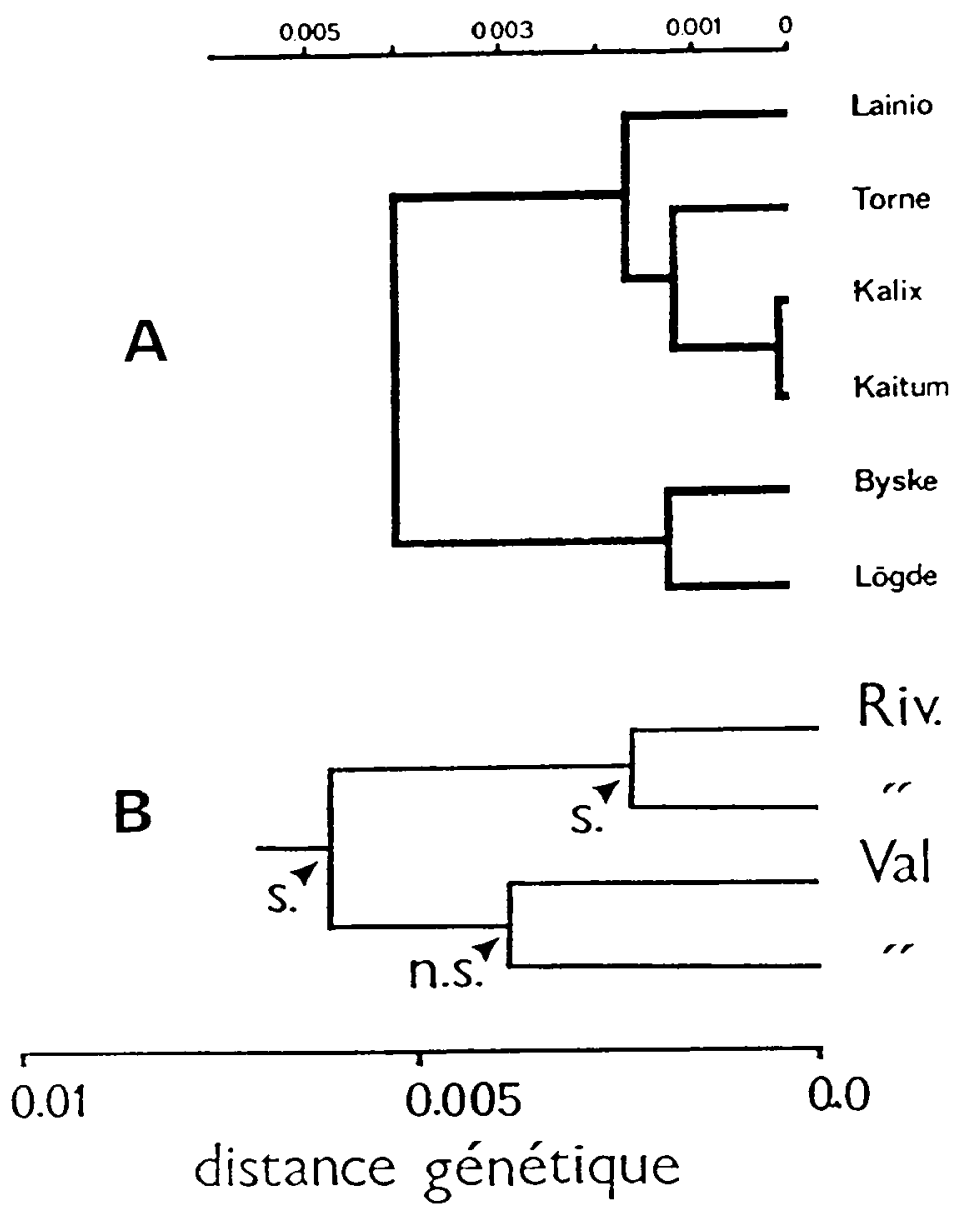

Riv. Chateau anadrome " sédentaire Val Studer anadrome " " sédentaire

figure $8 \quad$ : Deux exemples de différenciation entre populations de salmonidés.

A Salmo salar en Suède (RYMAN, 1981)

B Salmo trutta aux lles Kerguelen (Introduction unique en 1950, GUYOMARD et al., 1984).

figure $8 \quad$ : Two examples of genetic differentiation between salmonids populations.

A Salmo salar in Sweden (RYMAN, 1981)

B Salmo trutta in Kerguelen Islands (populations resulting from the same introduction around 1950, GUYOMARD et al., 1984).

ments intraspécifiques sont plus réduits et non systématiques (voir notamment FRANKEL et SOULE, 1981, p. 152 pour une discussion) et les essais réalisés notamment entre les groupes atlantiques et méditerranéens de truite fario (différenciation d'environ $10 \%$ ) ne semblent pas conduire à de tels phénomènes. De même chez la truite fardée (Salmo clarkii) l'existence dans la nature, à la suite de repeuplements, d'individus hybrides entre les différentes populations, voire avec la truite arc-en-ciel, espèce voisine, indique que la viabilité de ces hybrides n'est pas notablement inférieure, même si des mesures plus fines mettent en évidence de légères dépressions pour certains caractères (LEARY et al. 1985b; FERGUSON et al. 1988). A l'inverse, ces croisements peuvent révéler des performances intéressantes: c'est l'effet d'hétérosis (vigueur hybride) illustré notamment chez la carpe (WOHLFARTH et al. 1975) et le poisson-chat américain (PLUMB et al. 1975). Il faut donc concevoir les relations entre différenciation et performances des croisements selon un modèle de courbe en cloche ("optimum outcrossing distance" de BATESON, 1978) qu'il conviendrait d'étalonner dans chaque cas.

- Les individus de seconde génération peuvent à leur tour présenter des problèmes de viabilité. même lorsque la première génération s'est révélée viable, voire plus performante que les populations parentales. Ce phénomène peut résulter notamment de la formation d'anomalies chromosomiques lorsque les chromosomes de deux populations présentent un certain nombre de différences structurelles. Dans ce cas extrême, les individus de première génération peuvent 
être viables mais stériles et aucun individu de seconde génération n'est produit. On peut certes espérer voir disparaître progressivement les individus les moins viables sous l'effet de la sélection naturelle ou artificielle mais certains modèles théoriques illustrent la possibilité d'instaurer une dépression durable de la fertilité des individus à l'issue de tels croisements. Là aussi les exemples sont à prendre en dehors des espèces aquatiques (FRANKEL et SOULE, 1981 , p. 152 pour une revue) mais suffisent à illustrer la nécessité de prendre en compte ce problème.

\section{PROPOSITIONS CONCRĖTES}

Face aux différents arguments évoqués précédemment, le gestionnaire se trouve donc face à une contradiction éventuelle entre l'intérêt de regrouper une large variabilité génétique et le risque d'être confronté à des phénomènes négatifs liés à un regroupement d'unités génétiques incompatibles. En outre le caractère relativement discret et différé dans le temps de ces phénomènes (apparition en deuxième génération) risque de les rendre difficilement diagnostiquables sans que leur impact ne soit négligeable pour autant. En restant dans le cas de possibilités expérimentales limitées, il nous apparaît souhaitable d'adopter une stratégie triple

- La constitution d'une "population de référence" à partir d'une population ou du regroupement de populations extrêmement proches (de l'ordre de quelques \%o), cette proximité étant en outre confirmée par des considérations géographiques ou historiques. Cette ou ces populations seront choisies parmi celles présentant un niveau élevé d'hétérozygotie.

- La constitution d'une "population synthétique expérimentale" à partir d'un choix plus vaste de populations modérément différenciées.

Ces deux unités seront entretenues de manière similaire et leurs performances de survie, croissance et fertilité évaluées pendant au moins deux ou trois générations, surtout si la domestication s'accompagne d'une sélection volontaire de certains caractères. A l'issue de cette période, l'une des deux unités pourra être eventuellement éliminée, en particulier si leurs performances diffèrent de manière marquée. Par contre, si les performances sont voisines, la conservation des deux unités éventuellement dans deux écloseries différentes, constituera une sécurité précieuse et permettra de développer éventuellement des stratégies de gestion et d'amélioration originales. II est clair qu'une telle "population synthétique" ne constitue qu'une réponse partielle au problème de la conservation génétique. En effet, dans l'état actuel des méthodes de la génétique, il n'est pas envisageable de recréer les composantes qui auront été mélangées dans la population synthétique. Une population synthétique constitue donc une "base de départ" pour une opération nouvelle beaucoup plus qu'une opération "réversible" de conservation. Elle pourrait être utilisée notamment pour peupler des milieux "vierges" où des capacités d'accueil auraient été restaurées et où l'introduction d'une population à base génétique large pourrait permettre une meilleure adaptation à long terme de la population.

- L'étude expérimentale des croisements entre populations représentatives des groupes fortement différenciés. Compte tenu de la nécessitè de poursuivre cette étude jusqu'à la deuxième génération, elle ne pourra porter que sur un nombre restreint de "couples" et devra être conduite en parallèle avec les deux premières opérations. Elle peut cependant permettre de déceler des effets d'hétérosis, d'identifier des caractéristiques originales utilisables ultérieurement et de préciser les limites des unités biogéographiques à protéger impérativement.

\section{GESTION GÉNÉTIQUE DES STOCKS}

Une fois constitué un stock en pisciculture, deux cas peuvent se présenter

- le stock ast "fermé", autrement dit, on souhaitera ne plus réaliser de nouvelles introductions dans cette population. Cette politique peut être justifiée notamment pour des raisons sanitaires, par des difficultés pratiques d'accès aux stocks naturels fondateurs ou par la volonté de mettre en place un programme de sélection au sein de la population pour lui conférer des caractéristiques originales;

- le stock reste "ouvert", et de nouvelles introductions à partir des stocks naturels sont prévues; il importe dans ce cas d'en définir les bases théoriques.

\section{LE CAS DES STOCKS FERMÉS}

\section{a) facteurs d'évolution}

Les processus pouvant conduire à l'évolution génétique d'un stock fermé ont été définis dans l'article de R. GUYOMARD : mutations et recombinaisons pourront créer de nouvelles combinai- 
sons génétiques, dérive et sélection modifieront la structure génétique de la population et conduiront éventuellement à une perte de variabilité. Notons que l'on désigne parfois à tort l'ensemble de ces processus d'évolution sous le terme de "dérive", alors que la dérive au sens strict n'est que l'un d'entre eux.

Nous nous placerons dans le cas où le gestionnaire du stock fermé souhaite conserver son stock "génétiquement proche" du stock naturel. C'est notamment le cas pour des stocks destinés au repeuplement. Cela implique donc:

- qu'il ne pratique pas de sélection volontaire pour modifier un caractère donné (dans le cas contraire, voir l'article de KRIEG et CHEVASSUS sur les schémas d'amélioration génétique);

- que ses méthodes d'élevage soient suffisamment maîtrisées pour éviter la mise en place d'une sélection naturelle "cachée" au sein de l'élevage. Cela suppose un taux de survie satisfaisant, les réductions d'effec if nécessaires se faisant "au hasard", et une bonne maîtrise de la maturation sexuelle en captivité, pour éviter l'entretien du stock avec les seuls individus qui "acceptent de se reproduire". (Dans le cas contraire, il sera préférable de renoncer à l'option de stock fermé, au moins pour un entretien à long terme).

Si ces conditions sont remplies, le principal facteur qui va gouverner l'évolution du stock sera la dérive génétique dont nous allons maintenant préciser les conséquences.

\section{b) Dérive et perte de variabilité génétique}

Comme pour la constitution initiale, l'entretien d'un stock fermé ne fera appel à chaque génération qu'à un nombre restreint de reproducteurs. On peut donc calculer comme dans la première partie un effectif efficace $N_{e}$ à chaque génération. Lorsque cet effectif est constant, la variabilité génétique résiduelle $V_{t}$ au bout de $t$ générations est donnée par:

$$
V_{1}=V_{0}\left(1-\frac{1}{2 N_{e}}\right)^{t}
$$

Vo étant la variabilité génétique initiale.

Lorsque l'effectif efficace varie d'une génération à l'autre, la formule précédente est valable en première approximation, l'effectif efficace $\mathbf{N}_{\mathbf{e}}$ étant égal à la moyenne harmonique des effectifs efficaces à chaque génération.

$$
\frac{1}{N_{e}}=\frac{1}{N_{1}}+\frac{1}{N_{2}}+\cdots+\frac{1}{N_{1}}
$$

La figure 9 illustre les conséquences de cette formule dans quatre situations différentes. Elle montre notamment :

- qu'il ne faut pas surestimer le problème de l'échantillonnage initial au détriment de l'entretien ultérieur:

- que des "goulots d'étranglement" répétés (exemple D) conduisent à une perte importante de variabilité, même lorsqu'ils sont séparés par des générations à fort effectif génétique:

- qu'une gestion rationnelle des stocks permet de minimiser le phénomène à moyen terme : ainsi, la création et l'entretien d'une population fermée avec une vingtaine de "couples" de reproducteurs, utilisés seulement à l'âge de 4 ans, permet au bout de 30 ans d'avoir conservé plus de $90 \%$ de la variabilité génétique initiale de la population.

Dans la pratique, l'étude du polymorphisme enzymatique permet de suivre l'évolution de la variabilité génétique au sein des populations, à condition de connaître leur variabilité initiale. C'est pourquoi les résultats du tableau ll doivent être interprétés avec prudence car ils ne constituent qu"une étude "instantanée" de populations non suivies depuis leur fondation.

\section{c) Dérive et consanguinité}

L'une des conséquences de la gestion de populations fermées d'effectif limitè est l'apparition d'un certain niveau de consanguinité au sein de la population. 


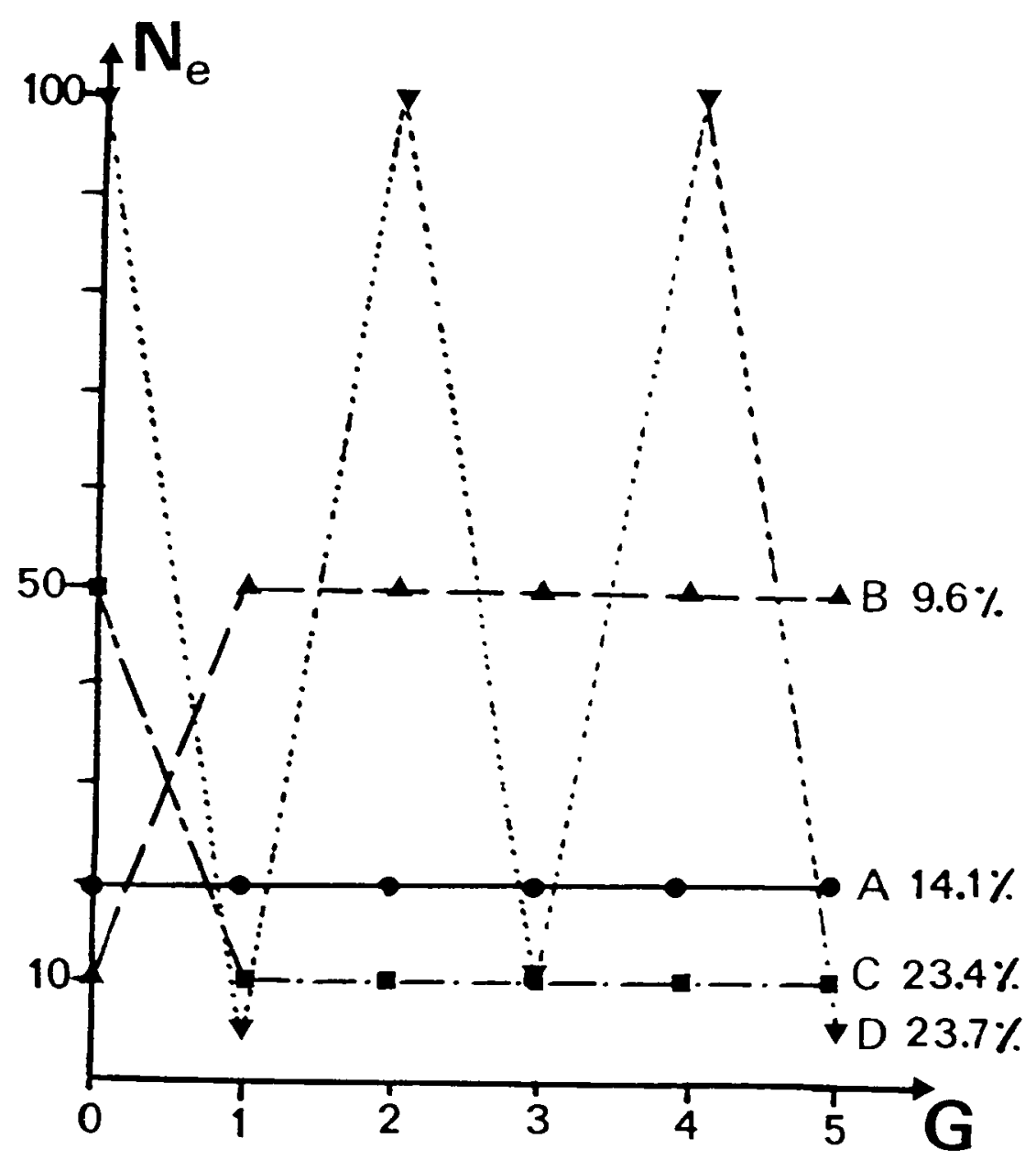

figure 9 : Perte de variabilité après 5 générations pour diverses évolutions de l'effectif efficace.
A Effectif constant $\mathrm{N}_{\mathrm{e}}=\mathbf{2 0}$
$B$ effectif fondateur faible (10) puis $\mathrm{N}_{\mathrm{e}}=\mathbf{5 0}$
C Effectif fondateur important (50) puis $\mathrm{N}_{\mathrm{e}}=10$
D Effectif fluctuant de $\mathbf{N}_{\mathbf{e}}=100$ à $\mathbf{N}_{\mathbf{e}}-5$

figure 9

Loss of genetic variability after 5 generations for different evolutions of effective number

A Constant effective number $\mathrm{N}_{\mathrm{e}}=20$

$B$ Low initial number (10) then $\mathrm{N}_{\mathrm{e}}=50$

C High initial number $(50)$ then $\mathrm{N}_{\mathbf{e}}=10$

D Effective number fluctuations between 5 and 100

Le coefficient de consanguinité $F$, défini comme la probabilité que les deux gènes d'un individu soient issus d'un ancêtre commun, est en effet directement relié à la perte de variabilité génétique par la formule :

$$
F t=1-\frac{V_{t}}{V_{0}}
$$

Cette augmentation de consanguinité se traduit généralement par une baisse notable des performances des individus. La figure 10 en donne un exemple concernant la croissance des salmonidés. Pour une augmentation de consanguinité de $1 \%$, la croissance est déprimée d'environ 0,6 à $0,8 \%$. 


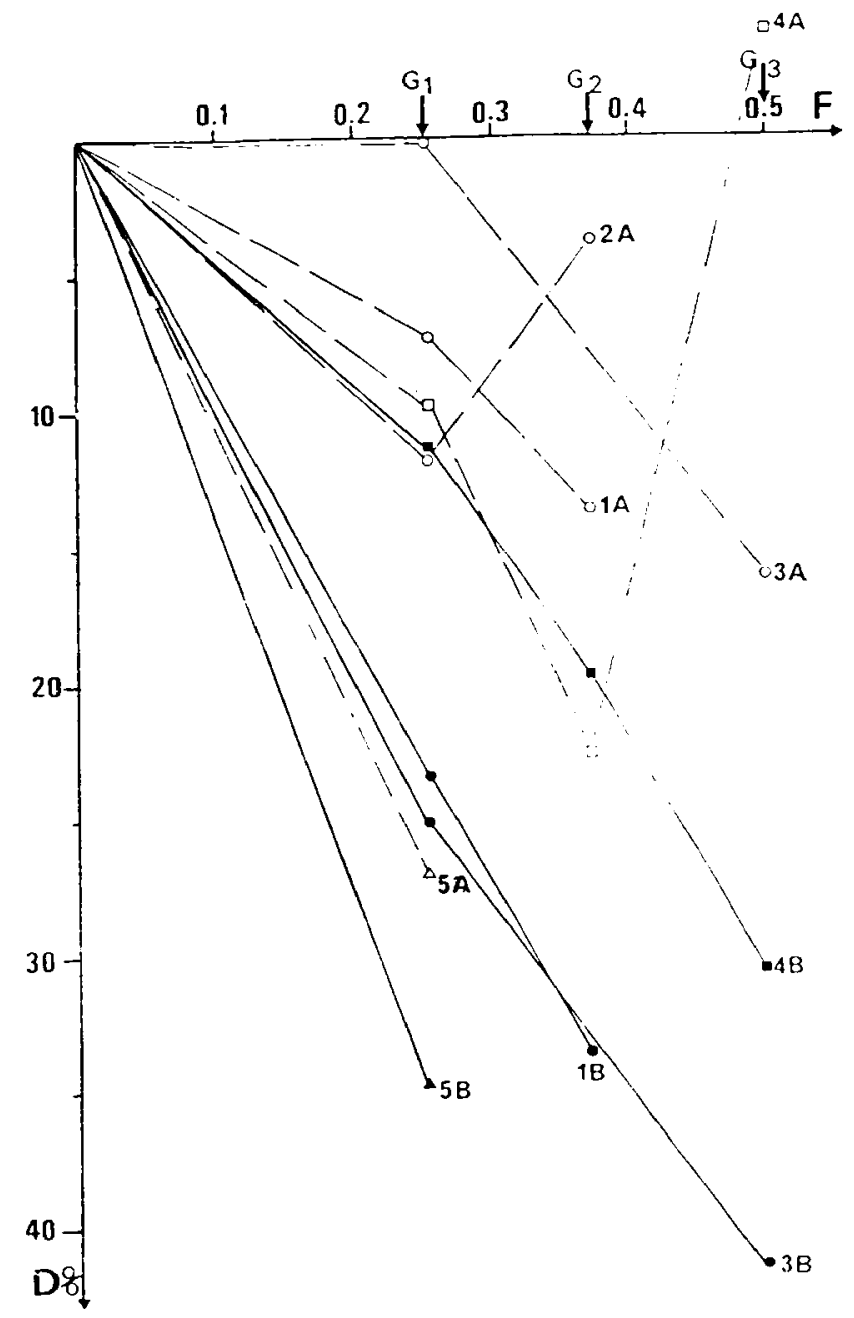

figure 10 : Dépression de la croissance en fonction du coefficient de consanguinité $F$ chez les salmonidés.

A Croissance précoce (à l'âge d'environ 6 mois)

B Croissance tardive (à l'àge de 1 à 3 ans).

figure 10 : Growth depression as a function of inbreeding coefficient $F$ in salmonids.

A Early growth (6 months of age)

B Later growth (1 to 3 years of age)

1 \& 2 Kincaid, 1976 Salmo gairdneri

3 Kincaid, 1983 Salmo gairdneri

4 Gjerde et al., 1983 Salmo gairdneri

5 Cooper, 1961 Salvelinus fontinalis.

Pour limiter ces effets de consanguinité, plusieurs mesures sont envisageables

- augmenter l'effectif efficace (voir paragraphe précédent);

- opérer une "contre sélection" en ne conservant que les individus les plus performants pour les critères affectés. Cette solution doit être utilisée avec prudence; d'autre part elle ne permettra en theorie que de corriger les effets sur les caracteres les plus facilement observables. Lorsqu'il s'agit de populations destinées au repeuplement, il ne sera donc pas possible en particulier de corriger les effets délétères éventuels sur le comportement en milieu naturel. D'autre part, cette sélection peut conduire à augmenter la perte de variabilité, soit en réduisant davantage l'effectif reproducteur, soit en conservant préférentiellement des individus "génétiquement proches" au lieu d'individus tirés au hasard;

- modifier le plan de croisement des reproducteurs; en effet les formules précédentes sont valables lorsque l'appariement des reproducteurs se fait au hasard. Lorsque l'on connaît la généalogie des individus, il est possible d'élaborer des schémas d'accouplement minimisant le 
progrès de la consanguinité. Cependant il est gènéralement difficile de conserver cette information généalogique chez les poissons, du fait des difficultés d'un marquage individuel précoce On peut dans ce cas avoir recours à la création de sous-groupes utilisés dans un système de croisement "rotatif" (figure ou KINCAID 1977). On peut également scinder la population en deux sous-populations entretenues indépendamment et produisant à chaque génération des individus "croisés" destinés à être "exportés". Cette stratégie est rendue possible par la grande fécondité des poissons. Comme la dérive dépend beaucoup plus du nombre de reproducteurs utilisés que du nombre de descendants par reproducteur, on peut scinder la ponte de chaque femelle en deux parties

- la grosse majorité des œufs sera fécondée par des mâles de l'autre sous-population. Les produits obtenus seront donc non consanguins et fourniront les individus destinés à la production de sujets de repeuplement :

- une petite partie sera fécondée par des mâles de la même sous-population et servira à l'entretien de cette sous-population. La consanguinité évoluera certes plus rapidement au sein de cette sous-population que dans une population brassant tous les reproducteurs mais les effets de cette consanguinité seront limités aux sujets maintenus en captivité.

\section{d) Dérive et modification des fréquences géniques}

Une autre manifestation possible de la dérive est l'évolution aléatoire des fréquences géniques. La figure 11 donne un exemple de ce phénomène. Pour deux systèmes enzymatiques différents, on observe dans un cas (CPKi) que l'allèle le plus fréquent a vu sa fréquence diminuer alors que le phénomène est inverse pour l'autre système (AGP2). II en résulte globalement une disjonction importante entre les stocks naturels et les stocks domestiques qui sont censés les "soutenir".

Les méthodes pour limiter ce phénomène sont les mêmes que celles évoquées pour réduire la perte de variabilité génétique ou l'évolution de la consanguinité.

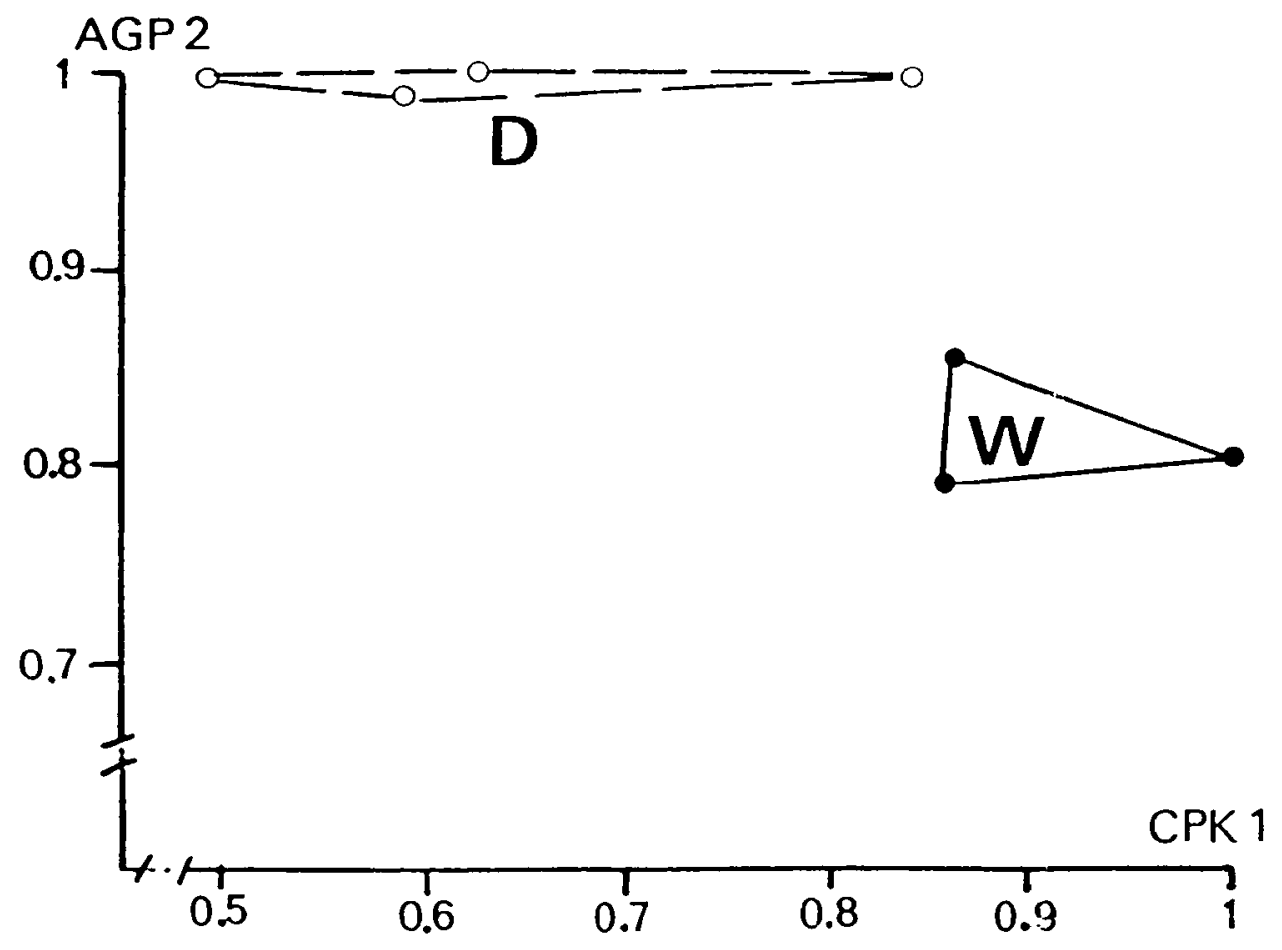

figure 11 : Comparaison des fréquences de l'allèle majoritaire pour 2 locus dans 3 populations sauvages suédoises de truite fario (Salmo trutta) de la rivière Skellefteälven (W) et dans 4 stocks d'écloserie (D) fondés à partir de reproducteurs de cette rivière (d'après RYMAN, 1981).

figure 11 : Comparison of the frequencies of the most common allele for two loci in 3 wild populations (W) of brown trout (Salmo trutta) from the River Skellefteälven (Sweden) and in 4 hatchery stocks (D) founded with spawners from this river (from RYMAN, 1981). 


\section{LE CAS DES STOCKS OUVERTS}

Si la gestion rationnelle de stocks fermés peut permettre à moyen terme de les maintenir proches des populations naturelles constitutives, il est cependant clair que des échanges génétiques avec ces populations naturelles peuvent constituer un facteur d'homogénéisation complémentaire auquel il peut être souhaitable de recourir.

\section{a) aspects théoriques}

Soit une population captive d'effectif génétique $\mathrm{Ne}$ issue d'une population naturelle supposée de grande taille. Nous supposerons qu'à chaque génération $K$ individus issus de la population $\mathrm{K}$

naturelle sont introduits dans la population captive. On désignera par $m=\frac{K}{N_{e}}$ le taux de renouvellement de la population captive.

La frèquence f d'un allèle donné dans la population captive va évoluer sous l'effet de deux phénomènes:

- la dérive liée à la taille limitée de la population,

- l'introduction d'animaux à chaque génération.

On montre que si fo est la fréquence de l'allèle dans la population naturelle, la fréquence f ne s'écartera en moyenne que d'une valeur:

$$
f-f_{0}=2 \sqrt{\frac{f(1-f)}{1+4 \mathrm{mN}_{\theta}}}
$$

(CROW et KIMURA, 1970)

On constate donc que cet écart dépend uniquement du produit $N_{e} m$, autrement dit, de l'effectif $\mathrm{K}$ d'individus migrant d'une population à l'autre, quelle que soit la taille de la population captive.

Ainsi pour un effectif $K=10$ et un allèle relativement fréquent ( $f=0,7$ ), l'écart restera en moyenne d'environ 0.14 . II suffit donc en théorie d'un nombre restreint d'individus migrants pour assurer une relative homogénéité génétique entre une population d'élevage et une population naturelle, quelle que soit la taille de la population d'élevage.

Une autre conséquence de la migration est de limiter l'évolution de la consanguinité. En effet, au lieu d'évoluer de manière continue comme dans le cas d'une population fermée, le coefficient de consanguinité atteint une valeur d'équilibre:

$$
F=\frac{1}{1+4 \mathrm{mN}_{\theta}}
$$

On voit également qu'un nombre faible de migrants suffit à limiter considérablement l'évolution de la consanguinité. Ainsi pour une population fermée d'effectif $N_{e}=50$, la consanguinité évoluera d'environ $1 \%$ par génération. Si l'on introduit $\mathrm{K}=\mathrm{mN}_{\mathrm{e}}=5$ nouveaux animaux à chaque génération, elle restera à une valeur d'équilibre d'environ $5 \%$.

\section{b) Aspects pratiques}

Dans la pratique, ce flux génétique peut être assuré en prélevant des reproducteurs ou des gamètes mâles ou femelles dans la population naturelle. II importe cependant de vérifier que cette opération conduit bien à apporter un réel flux génétique vers la population d'élevage. En effet de nombreux phénomènes peuvent réduire l'effet de cette pratique :

- les reproducteurs peuvent présenter une faible survie en captivité

- la qualité des gamètes prélevés peut être médiocre, notamment du fait de l'ignorance de la date d'ovulation des femelles:

- les alevins produits peuvent présenter une croissance et une survie plus faibles. La figure 12 illustre un exemple de ce phenomene dans le cas d'un croisement entre une souche d'une 


\begin{tabular}{l|l|l}
\hline$\sigma$ & $G O$ & EL \\
\hline$G 0$ & $\mathrm{~s}=90,4$ & $\mathrm{~s}=60,2$ \\
$\mathrm{p}=4,38$ & $\mathrm{p}=3,19$ \\
\hline$E L$ & $\mathrm{~s}=63,6$ & $\mathrm{~s}=29,2$ \\
$\mathrm{p}=3,65$ & $\mathrm{p}=1,99$ \\
\hline
\end{tabular}

A

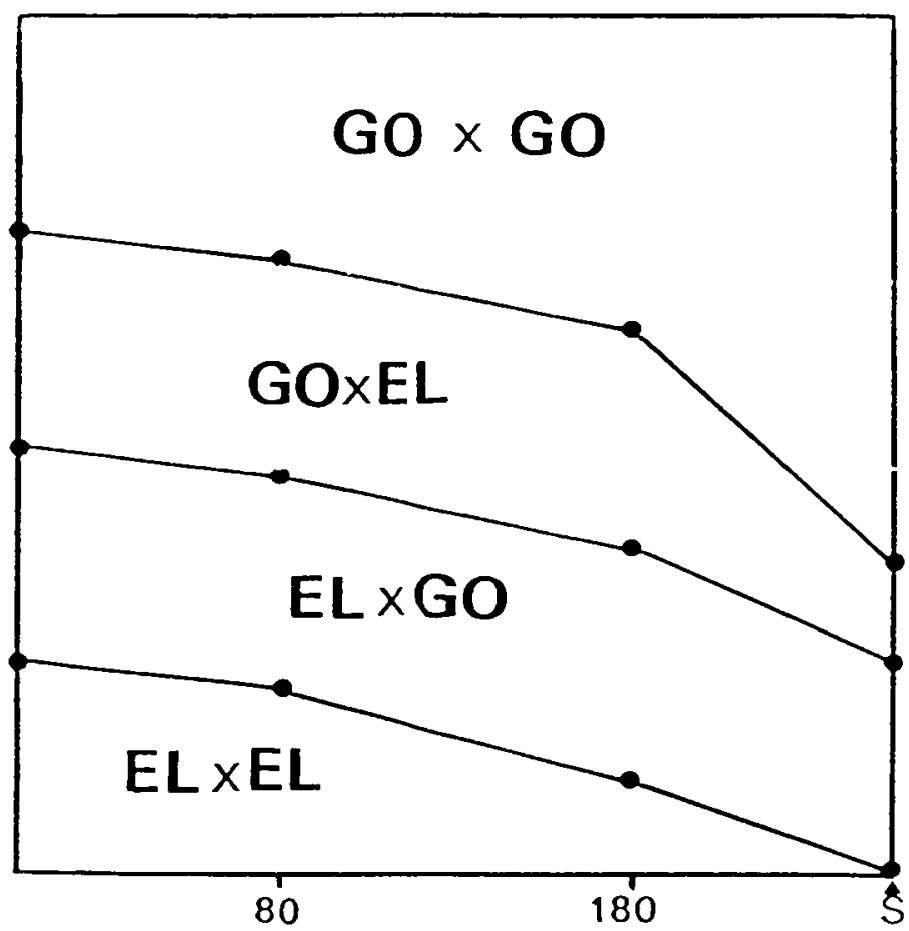

B

figure 12 : A Taux de survie $s$ et poids moyen $p$ à 180 jours de 4 groupes de truites fario Salmo trutta issues du croisement d'une souche domestique GO et d'une souche sauvage EL.

B Simulation de l'évolution de la proportion des 4 génotypes en fonction de la survie à 80 et 180 jours et d'une sélection $S$ éliminant à $180 \mathrm{~J}$ les animaux de moins de $4 \mathrm{~g}$ (63\% d'élimination) (d'après GUYOMARD, 1989).

figure 12 : A Survival rate $s$ and mean weight $p$ at day $\mathbf{1 8 0}$ for $\mathbf{4}$ groups of brown trout resulting from crosses between a domestic strain GO and a wild strain EL.

$B$ Evolution of the proportion of the $\mathbf{4}$ groups as a result of survival rate at 80 and 180 days and after a selection $S$ eliminating the smallest animals (less than $4 \mathrm{~g}$, $63 \%$ of elimination) (from GUYOMARD, 1989).

pisciculture de repeuplement bretonne $(G O)$ et des animaux sauvages issus de l'ELORN Si les 4 groupes sont mélangés, les effets combinés de la plus faible survie et d'un tri sur la croissance peuvent éliminer presque totalement les individus issus de la souche sauvage.

Nous suggérons donc d'organiser le flux génique en deux temps

- dans un premier temps, on réalisera un croisement entre des femelles domestiques et des mâles sauvages. Ceci permet de disposer d'ovules de qualité connue et de ne pas ponctionner inutilement les stocks naturels, le sperme pouvant être prélevé sur les mâles in situ. Ce lot "hybride" sera élevé jusqu'au stade marquable. Pendant cette période, on pourra donc observer la survie et la croissance de ces animaux et pratiquer en outre d'éventuels examens sanitaires: 
- dans un deuxième temps, integration d'un nombre donné de ces mâles et femelles hybrides dans le stock de reproducteurs de la population domestique.

Cette opération en deux temps est un peu plus complexe mais présente de bien meilleures garanties de réussite. Elle peut d'ailleurs n'être réalisée que de manière espacée, la population étant entre-temps gérée comme une population fermée.

\section{CONCLUSION GÉNÉRALE}

Pour des populations naturelles, le recours à l'élevage est souvent considéré comme un "abatardissement" irrémédiable. Ce mode de pensée est sans doute en partie influencé par les observations sur les mammifères captifs, où la transmission "culturelle" (en particulier par la mère) de certains comportements peut jouer un rôle important dans l'adaptation du jeune au milieu naturel. La rupture du lien avec le milieu naturel peut donc dans ce cas effectivement conduire à une perte difficilement réversible d'adaptation. Il est cependant peu probable que de tels phénomènes puissent jouer chez les poissons un rôle notable. Une autre cause d" "abatardissement" peut être constituée par le développement de pathologies facilitées par l'élevage intensif et transmissibles à la descendance. Ce phénomène ne doit pas être négligè et justifie la mise en place de suivis sanitaires précis dans ces élevages. Reste l'abatardissement au sens génétique du terme. Le présent papier avait pour but de mieux en préciser les mécanismes et de montrer que des mesures simples pouvaient à moyen terme (10-20 ans) en limiter considérablement la portée. Il est donc possible à notre avis de faire naitre à partir de reproducteurs maintenus en captivité depuis plusieurs générations des sujets ayant conservé des caractéristiques génétiques proches des sujets "sauvages". II convient bien sûr ensuite de définir leur mode d'élevage et d'utilisation, mais c'est là un tout autre sujet.

\section{RÉFÉRENCES}

ALLENDORF F.W. and LEARY R.F., 1988. Conservation and distribution of genetic variation in a polytypic species, the cutthroat trout. Conservation Biology, 2, 170-184.

ALLENDORF F.W., RYMAN N., STENNEK A. and STAHL G., 1976. Genetic variation in Scandinavian brown trout (Salmo trutta) : evidence of distinct sympatric populations. Hereditas, 83, 73-82.

AYALA F.J., 1965. Relative fitness of populations of Drosophila serrata and Drosophila birchii. Genetics, 51, 527-544.

BATESON P., 1978. Sexual imprinting and optimal outbreeding. Nature, 273, 659-660.

BEACHAM T.D. and WITHLER R.E., 1985. Heterozygosity and morphological variability of chum salmon (Oncorhynchus keta) in southern British Columbia. Heredity, 54, 313-322.

CHEVASSUS B., 1983. Hybridization in fishes. Aquaculture, 33, 245-262.

COOPER E.L., 1961. Growth of wild and hatchery strains of brook trout. Trans. Am. Fish. Soc., 90. 424-438.

CROSS T.F. and KING J., 1983. Genetic effects of hatchery rearing in Atlantic salmon. Aquaculture, 33, 33-40.

CROW J.F. and KIMURA M., 1970. An Introduction to population genetics theory. Harper and Row, publishers. New-York.

DANZMANN R.G., FERGUSON M.M., ALLENDORF F.W. and KNUDSEN K.L., 1986. Heterozygosity and developmental rate in a strain of rainbow trout (Salmo gairdneri). Evolution, 40, 86-93.

FERGUSON M.M., DANZMANN R.G. and ALLENDORF F.W., 1985. Developmental divergence among hatchery strains of rainbow trout (Salmo gairdneri). I. Pure strains. Can. J. Genet. Cytol., 27, 289-297.

FERGUSON M.M., DANZMANN R.G. and ALLENDORF F.W., 1988. Developmental success of hybrids between two taxa of salmonid fishes with moderate structural gene divergence. Canadian Journal of Zoology, 66, in press

FOLTZ D.W., NEWKIRK G.F. and ZOUROS E., 1983. Genetics of growth rate in the American oyster: absence of interactions among enzyme loci. Aquaculture, 33, 157-165.

FRANKEL O.H. and SOULE M.E., 1981. Conservation and evolution. Cambridge University Press.

GJERDE B., GUNNES K. and GJEDREM T., 1983. Effect of inbreeding on survival and growth in rainbow trout. Aquaculture, 34, 327-332

GUYOMARD R., 1981. Electrophoretic variation in four french populations of domesticated rain bow trout (Salmo gairaneri). Can. J. Genetic. Cytol., 23, 33-47. 
GUYOMARD R., 1987. Différenciation génétique des populations de saumon atlantique : revue et interprétation des données électrophorétiques et quantitatives. M. THIBAULT et R. BILLARD, ed. Restauration des rivières à saumons, colloque Bergerac. INRA, 298-308.

GUYOMARD R., 1989. Diversité génétique et gestion des populations naturelles de truite commune. Colloque sur la truite commune, Le Paraclet (France), septembre 1988, (sous presse).

GUYOMARD R., GREVISSE C., OURY F.X., DAVAINE P., 1984. Evolution de la variabilité génétique inter et intrapopulation de populations de salmonidés issues de mêmes pools géniques. Can. J. Fish. Aquat. Sci., 41, 1024-1029.

HINDAR K., RYMAN N. and STAHL G., 1988. Genetic differentiation among local populations and morphotypes of arctic charr, Salvelinus alpinus. Biol. J. Linn. Sic. (in press).

JONES L.P., FRANKHAM R., BARKER J.S.F., 1968. The effect of population size and selection intensity in selection for a quantitative character in Drosophilia. II. Long term response to selection. Genet. Res., 12, 249-266.

KIMURA M. and CROW J.F., 1963. The measurement of effective population number. Evolution, 17, 279-288.

KINCAID H.L, 1976 b. Inbreeding in rainbow trout (Salmo gairdneri). J. Fish. Res. Board Can., 33 , 2420-2426.

KINCAID H.L., 1977. Rotational line crossing : an approach to the reduction of Inbreeding accumulation in Trout Brood stocks. Prog. Fish. Cult., 39, 179-181.

KINCAID H.L., 1983, Inbreeding in fish populations used for aquaculture. Aquaculture, 33, $215-227$.

KOEHN R.K., DIEHL W.J. and SCOTT T.M., 1988. The differential contribution by individual enzymes of glycolysis and protein catabolism to the relationship between heterozygosity and growth rate in the coot clam., Mulinia lateralis. Genetics, 118, 121-130.

LACAVA J. and HUGHES J., 1984. Determining minimum viable population levels. Wild Soc. Bul., 12, 370-376.

LEARY R.F., ALLENDORF F.W. and KNUDSEN K.L., 1983. Developmental stability and enzyme heterozygosity in rainbow trout. Nature, 301, 71-72.

LEARY R.F., ALLENDORF F.W. and KNUDSEN, 1984. Superior developmental stability of enzyme heterozygotes in salmonid fishes. American Naturalist, 124, 540-551.

LEARY R.F., ALLENDORF F.W. and KNUDSEN K.L., 1985 a. Developmental instability as an indicator of reduced genetic variation in hatchery trout. Transactions of the American Fisheries Society, 114, 230-235.

LEARY R.F., ALLENDORF F.W. and KNUDSEN K.L., 1985 b. Developmental instability and high meristic counts in interspecific hybrids of salmonid fishes. Evolution, 39, 1318-1326.

LERNER J.M., 1954. Genetic homeostasis. Dover Publications, New York.

MC ANDREW B.J., WARD R.D. and BEARDMORE J.A., 1986. Growth rate and heterozygosity in the plaice, Pleuronectes platessa. Heredity, 57, 171-180.

MITTON J.B., 1978. Relationship between heterozygosity for enzyme loci and variation of morphological characters in natural populations. Nature, 273, 661-662.

NEI M., 1975. Molecular population genetics and evolution. North Holland, Amsterdam and New York, $228 \mathrm{pp}$.

NEI M., 1978. Estimation of average heterizygosity and genetic distance from a small number of individuals. Genetics, 89, 583-590.

PLUMB J.A., GREEN O.I., SMITHERMAN R.O. and PARDUE G.B., 1975. Channel catfish virus experiments with different strains of channel catfish. Trans. Am. Fish Soc. 104, 140-143.

RYMAN N., 1981 b. Conservation of genetic resources : experiences from the brown trout ( $S a l m o$ trutta). In : N. RYMAN (Editor), Fish Gene Pools. Ecol. Bull. (Stockholm), 34, 61-74.

STAHL G., 1981. Genetic differentiation among natural populations of atlantic salmon (Salmo salar) in northern Sweden. Ecol. Bull. (Stockholm), 34, 95-105.

STAHL G., 1983. Differences in the amount and distribution of genetic variation between natural populations and hatchery stocks of Atlantic salmon. Aquaculture, 33, 23-32.

WOHLFARTH G., MOAV R. and HULATA G., 1975. Genetic differences between the Chinese and the European races of the common carp. II. Multicharacter variation : a response to the diverse methods of fish cultivation in Europe and China. Heredity, 34, 341-350.

ZOUROS E. and FOLTZ D.W., 1987. The use of allelic isozyme variation for the study of heterosis. Isozymes: Current Topics in Biological and Medical Research, 13, 1-59. 